\title{
Polysaccharide Derivatives as Unique Chiral Selectors for Enantioselective Chromatography
}

\author{
Eric R. Francotte* \\ KGF-SCS Senior Industrial Investigator Award 2016
}

\begin{abstract}
From the beginning of the 1980s, the life science industry increasingly recognized the importance of chirality in biological interaction processes, but the methods for preparing optically pure drugs were still limited. Most of the syntheses of chiral compounds were performed starting from optically active building blocks (chiral pool), mainly from natural sources, or by resolution of the enantiomers via formation of diastereomers. In this context, there was a growing interest for enantioselective processes, such as synthetic methodologies and separation techniques for accessing optically pure substances in an effective manner. Among the separation approaches, enantioselective chromatography looked very promising and a project aiming to explore this option was started in the Central Research Laboratories of former Ciba-Geigy. This article reviews the story of this development which culminated in the discovery of highly efficient polysaccharide-based chiral stationary phases which have now become the gold standard in the world of enantioselective chromatography. It shows also how the technique of enantioselective chromatography has evolved from an analytical tool to a practical preparative technology, up to production scale.
\end{abstract}

Keywords: Enantioselective chromatography · Stereoisomer discrimination

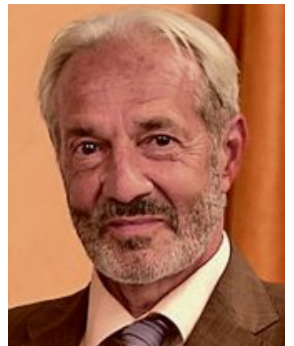

Eric Francotte joined the Central Research Laboratories of former CibaGeigy (Basel, Switzerland) in 1980 after completing a PhD in organic chemistry at the University of Louvain in Belgium (Prof. H.G. Viehe), and a postdoc at the University of Geneva (Prof. W. Oppolzer).

${ }^{\star}$ Correspondence: Dr. E. Francotte FrancotteConsulting, Orisstrasse 4, CH-4412 Nuglar (Formerly Ciba-Geigy, Novartis)

E-mail: eric.francotte@francotteconsulting.ch
For 10 years, he focused his research interest on the development of new chiral stationary phases for enantioselective separations of chiral compounds and worked in close collaboration with chemists in drug and agrochemical research. In 1991, he moved to the pharmaceutical division of Ciba, which became Novartis in 1996, where he was executive director until his retirement in 2016. He is now independent scientific advisor in the field of Separation Sciences. During his industrial research career as a project leader and section head, he developed and applied innovative chiral materials for the separation of enantiomers of numerous biologically active substances on an analytical and preparative scale, facilitating and accelerating the development of new drugs. The novel chiral stationary phases that he developed, have been commercialized and are now the gold standard in this field. He was also a pioneer for the implementation of new preparative chromatographic technologies such as simulated-moving-bed and supercritical fluid chromatography in the pharmaceutical industry. He received several awards for his contribution in the field of enantioselective chromatography, notably the Ciba Fellow Award (1995), the Jaubert Prize of the Geneva University (1998), and the Novartis Distinguished Scientist award (2000). He holds about 120 publications and patents.

\section{Introduction}

In 1980, no single chiral stationary phase was commercially available for enantioselective chromatography and a project aiming to develop optically active polymers to be used for the resolution of racemic drug and drug intermediates by chromatography was initiated in the central research laboratories of formerly Ciba-Geigy. The primary motivation for this project was driven by the growing awareness of the importance of chirality on biological activity.

At that time, a number of initial works on this topic had already been performed by a few groups in the US, Europe and Japan, but most of the investigations were isolated and preliminary. These works include the very first liquid chromatography separation of enantiomers by Henderson and Rules in 1939[1] and the resolution of the racemic Tröger base on lactose by Prelog and Wieland in 1944 (Table 1). ${ }^{[2]}$ Almost 30 years later, Davankov in Russia introduced the concept of chiral ligand exchange chromatography ${ }^{[3]}$ and Blaschke in Germany developed optically active polyacrylamides as chromatographic support for enantioselective separation. ${ }^{[4]}$ Further earlier reports include the utilization of cellulose, starch, partially acetylated and triacetylated cellulose, and proteins as chiral stationary phases for enantioselective 
liquid chromatography, or amino acids for enantioselective gas chromatography. ${ }^{[5]}$ Interestingly, most of the very early studies were performed using polysaccharides.

But it was not before the early 1980 s that the topic of enantioselective chromatography attracted growing attention, especially under the impulse of the group of Okamoto in Japan, ${ }^{[6]}$ the groups of Pirkle ${ }^{[7]}$ and Armstrong in the US, ${ }^{[8]}$ Schurig[9] and König[10] in Germany, and Lindner in Austria. ${ }^{[11]}$ In the same period of time, we started to synthesize a broad variety of optically active polymers pursuing the goal to develop novel effective chiral stationary phases (CSPs) for separating stereoisomers. This article summarizes the work performed at Ciba-Geigy and later at Novartis in the framework of this project and the successful development of unique chromatographic CSPs which have been commercialized and are now recognized as the new gold standard in the field. Today, enantioselective chromatography has become the first technique for the separation of stereoisomers and is used worldwide from the analytical up to production scale. A broad variety of chiral stationary phases is available but more than $90 \%$ of the applications are performed using polysaccharide-based stationary phases, emphasizing the exceptional ability of this class of molecular system to recognize chirality of molecules. At a preparative scale, enantioselective chromatography can be considered as a pseudo catalytic process as the 'chiral auxiliary' is not consumed during the enantioselective separation process and is continuously recycled. Moreover, unlike for classical catalytic reactions, the chiral 'catalyst' (selector) does not need to be optically pure to reach a complete separation and obtain the pure enantiomers in enantioselective chromatography. A lower optical purity will affect the performance of separation, but not necessarily the purity of the isolated enantiomers.

\section{Oligo- and Polysaccharides in Enantioselective Separations}

Mono-, oligo- and polysaccharides are essential molecular assemblies in living organisms. Their function is extremely diverse, covering the skeletal structure of animal and plants, energy storage, cell protection and recognition, therapeutic activity, solubilizing systems, seed dispersal, etc. They are also very abundant across the entire world.

Moreover, saccharides are, beside amino acids, the most important basic chiral molecules in life and this characteristic confers them the ability to operate as chiral discriminators. This feature has made them extremely useful as a source of chemical
Table 1. Milestones in the development of enantioselective chromatography

- 1904: Willstätter, first attempts on wool and silk

- 1939: Henderson and Rule on lactose

- 1943: Lecoq on lactose

- 1944: Prelog and Wieland on lactose

- 1948: Dent on cellulose (paper)

- 1951: Kotake et al. on cellulose (paper)

- 1952: Dalgliesh on cellulose (paper)

- 1954: Krebs and Rasche on starch

- 1957: Musso on cellulose powder

- 1966: Lüttringhaus and Peters on acetylated cellulose

- 1968 : Kratchanov and Popova on polygalacturonic acid

- from 1975, growing research activities in the field of chiral stationary phases for liquid chromatography

- early 1980s, first 'chiral' columns appear on the market

substances for the elaboration of powerful chiral stationary phases for the enantioselective chromatographic separation of enantiomeric mixtures. Over the last 30 years, a broad range of oligo- and polysaccharide-based phases have been developed as chiral selectors for enantioselective gas, liquid, and electrochemical separation processes. The chiral selectors include small oligosaccharide, cyclodextrin, and polysaccharide derivatives (Fig. 1) as well as cyclofructose derivatives and glycopeptides. While the cyclodextrin-based selectors dominate the gas and electrophoretic application areas, the polysaccharidebased stationary phases (mainly derived from cellulose and amylose) dominate by far the liquid chromatography world in terms of applications.

\section{Enantioselective Liquid Chromatography on Polysaccharide-based Phases}

\section{Cellulose Triacetate}

During the initial phase of our project at Ciba-Geigy Central Research, we synthesized cellulose triacetate (Fig. 2) according to the process described by Hess and Ljubitsch in $1928^{[12]}$ and used in the pioneer works of Hesse and Hagel in 1973. [13]

A glass column was packed with the material and tested with a series of internal racemic drug samples. We rapidly noticed that cellulose triacetate exhibited interesting properties in terms of chiral recognition for a variety of drugs developed in Ciba. As already pointed out by Hess and Hagel, we

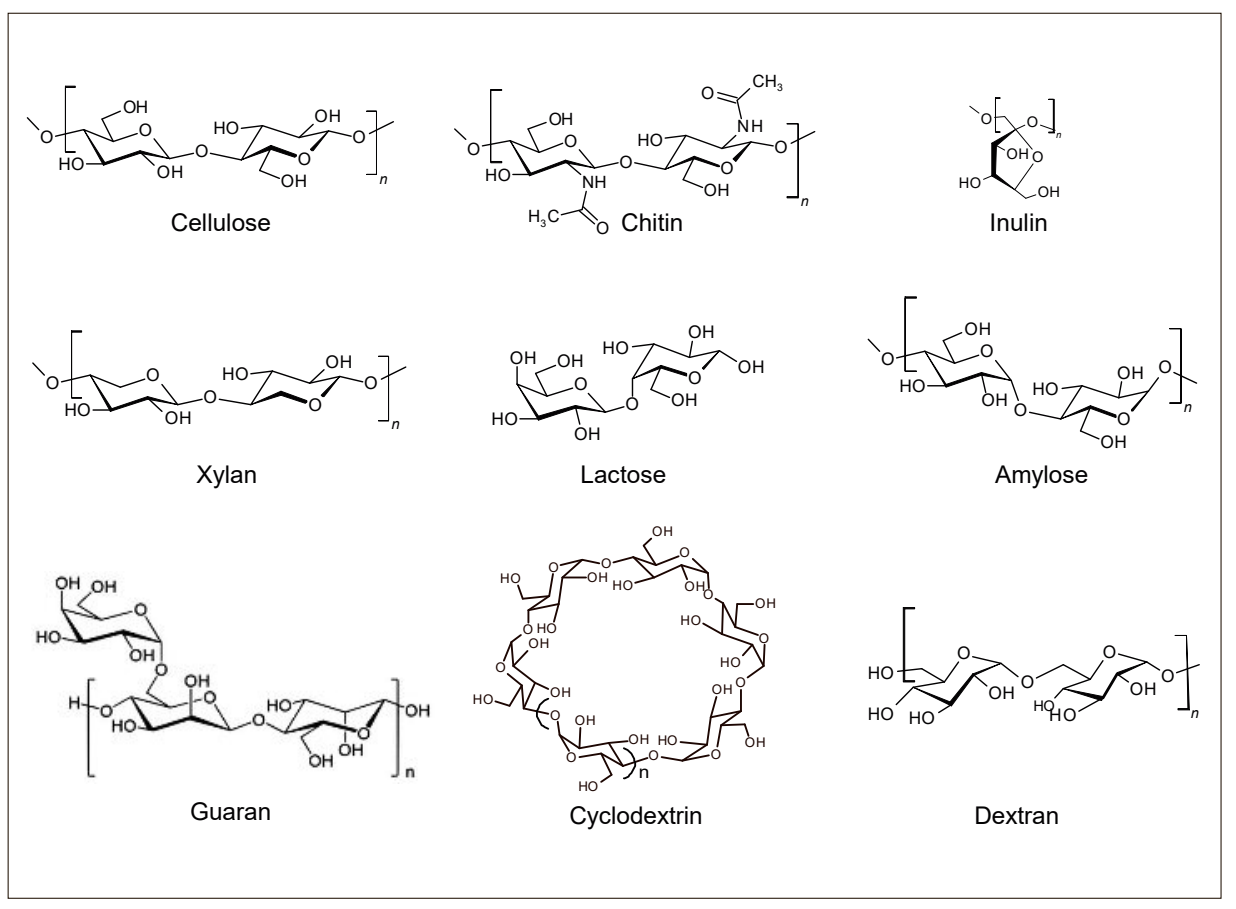

Fig. 1. Structures of oligo- and polysaccharides used in the course of our studies. 


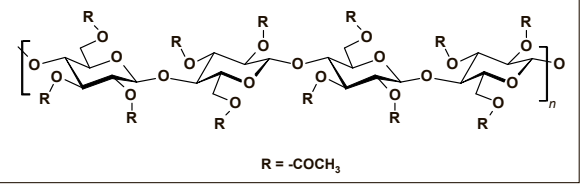

Fig. 2. Structure of cellulose triacetate.

confirmed that the preparation of cellulose triacetate under heterogeneous conditions was crucial to maintain the high chiral recognition ability of the material. The heterogeneous conditions were supposed to preserve the original micro-crystalline structure of cellulose. ${ }^{[13]}$ We intensely investigated this aspect ${ }^{[14]}$ and compared the chiral recognition ability of three different samples of cellulose triacetate (CTA): the first sample (CTA II) was obtained under homogeneous conditions of acetylation involving a dissolution and re-precipitation of CTA; the second sample (CTA I) was obtained under heterogeneous conditions, and the third sample (CTA I annealed) was prepared by annealing sample CTA I for $30 \mathrm{~min}$ at $240{ }^{\circ} \mathrm{C}$. X-Ray powder diffraction showed distinguished patterns for the three samples, indicating different crystal structures and degrees of crystallinity. As expected, annealed CTA I exhibits an enhanced degree of crystallinity compared to CTA I. Chromatography columns were packed with the three different CTA materials and their chiral recognition ability was compared using a set of ten racemic compounds. For all racemates, the best resolution was obtained on CTA I. Moreover, for a number of racemic compounds an inversion of elution order was observed between CTA I and CTA II (Fig. 3).

This result clearly demonstrated the importance of the supramolecular structure of CTA in the chiral recognition mechanism. Indeed, CTA I and CTA II possess the same chiral information at the molecular level but very different chiral recognition ability. It was concluded that the mutual arrangement of the individual polymeric chains in the crystal packing plays an essential role in the chiral discrimination process, by providing different chiral environments to the interacting solute. ${ }^{[14]}$ By analogy to the crystal forms I and II of native and regenerated cellulose respectively, the corresponding cellulose triacetates are designated as CTA I and CTA II. The difference between the two crystal forms is their parallel (form I) and anti-parallel (form II) arrangement of the cellulose chains in the crystal packing (Fig. 4).

This finding was fundamental in the context of the development of polysaccharide-based stationary phase for enantioselective chromatographic separations. The same phenomenon was observed later for other cellulose derivatives. ${ }^{[15]}$

Independently of this result, it was also empirically found that the chiral recognition of the solute by CTA could be often improved by achiral derivatization. Actually it was observed that molecules bearing a phenyl group and more particularly a nitrophenyl fragment generally showed a high affinity for CTA I and that a strong retention was mostly correlated with a good enantioselectivity. Based on this observation, we developed an achiral 'derivatization' strat- egy to solve enantiomeric separation requests which could not be achieved with the underivatized molecules. It was particularly useful for free alcohols, acids and amines. For acids, benzyl ester derivatives such as para-chloro or para-nitro were prepared. For amines and alcohols, para-nitrobenzamide and para-nitrobenzoate derivatives respectively were mostly well appropriate. A few typical examples of application of this approach are shown in Fig. 5.

This strategy was broadly exploited in our laboratories for at least a decade. At a time where no chiral stationary phase was commercially available, this was a very valuable strategy to extend the applicability of CTA I. The derivatization strategy has proven to be very useful for separating enantiomers of an extended diversity of chemical structures. Various applications exemplifying this strategy have been reported for numerous chiral building blocks and auxiliaries. ${ }^{[16]}$ In this report, cyclic and linear mono alcohols, diols, glycerol derivatives, epoxides and lactones were almost all easily resolved on CTA I into the respective enantiomers after simple derivatization of the racemic alcohols as para-substituted benzoylesters.

For example for racemic 2-trans-phenylcyclohexanol, which is not resolved on CTA I in the non-derivatized form, all the tested para-substituted benzoylester derivatives could be easily resolved on CTA I. ${ }^{[16]}$ This example shows also that both the retention and the enantioselectivity were strongly affected by the type of substituent in para-position of the phenyl ring, indicating a determining contribution of the

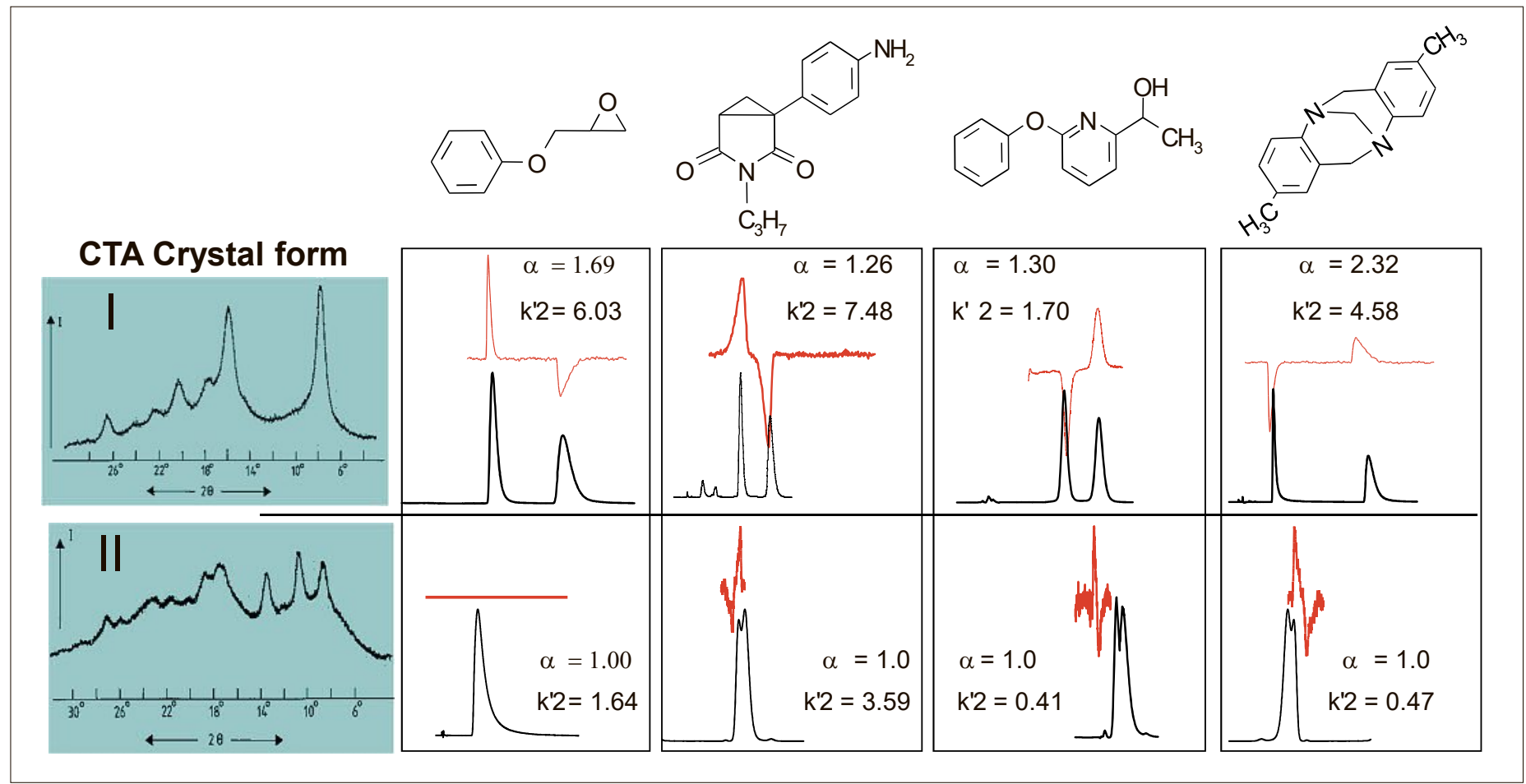

Fig. 3. Influence of the crystal form of cellulose triacetate on its chiral recognition ability. On the left, X-ray diffractograms of cellulose triacetate I and II 


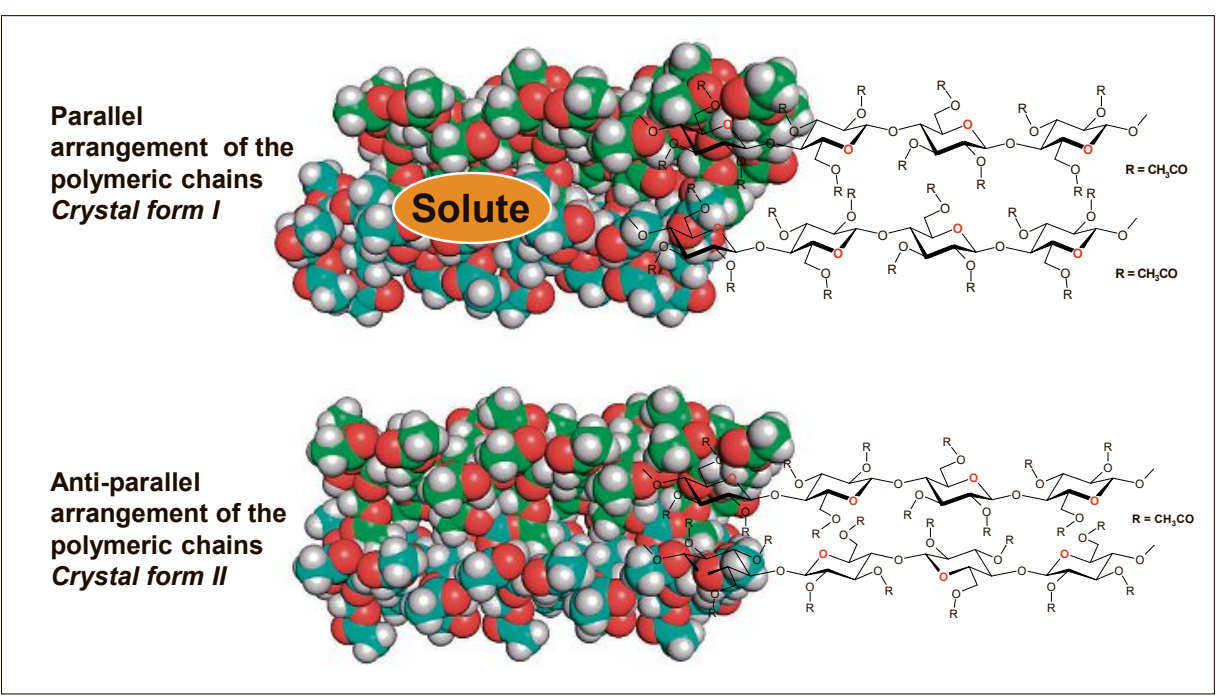

Fig. 4. Supramolecular arrangement of cellulose triacetate I and II.

derivatizing group in the chiral discrimination process with CTA I, even though this group is relatively remote from the chiral center. Moreover, it was observed that the influence of the substituent in para-position did not follow the same order for all substrates, suggesting that different interaction sites are present in CTA I, providing different spatial and electronic requirements. ${ }^{[16]}$ These two findings were important in the context of understanding the mechanism of 'chiral recognition' of CTA I. In particular the presence of multiple interaction sites in the polymer matrix of CTA I might explain the broad diversity of chiral substances which can be resolved by chromatography on this polymeric material. However, this feature emphasizes the difficulty to rationalize the use of the systematic derivatization of chi- ral compounds in order to improve their resolution. Nevertheless, the derivatization strategy was successfully applied on an empirical basis to solve a huge number of practical problems in the early phase time of the technology. By systematically applying this derivatization strategy, we could achieve a success rate of about $80 \%$ (40\% totally, $40 \%$ strongly enriched) for all chiral samples using the single CTA I stationary phase.

The same strategy was applied to determine the enantioselectivity of the pharmacokinetics of the enantiomers of the drug oxindanac in humans. ${ }^{[17]}$ This was one of the very first applications of direct chromatographic separation of enantiomers and the first using a polysaccharide-based CSP for pharmacological studies. In this case, the chiral lability of the acid (drug)

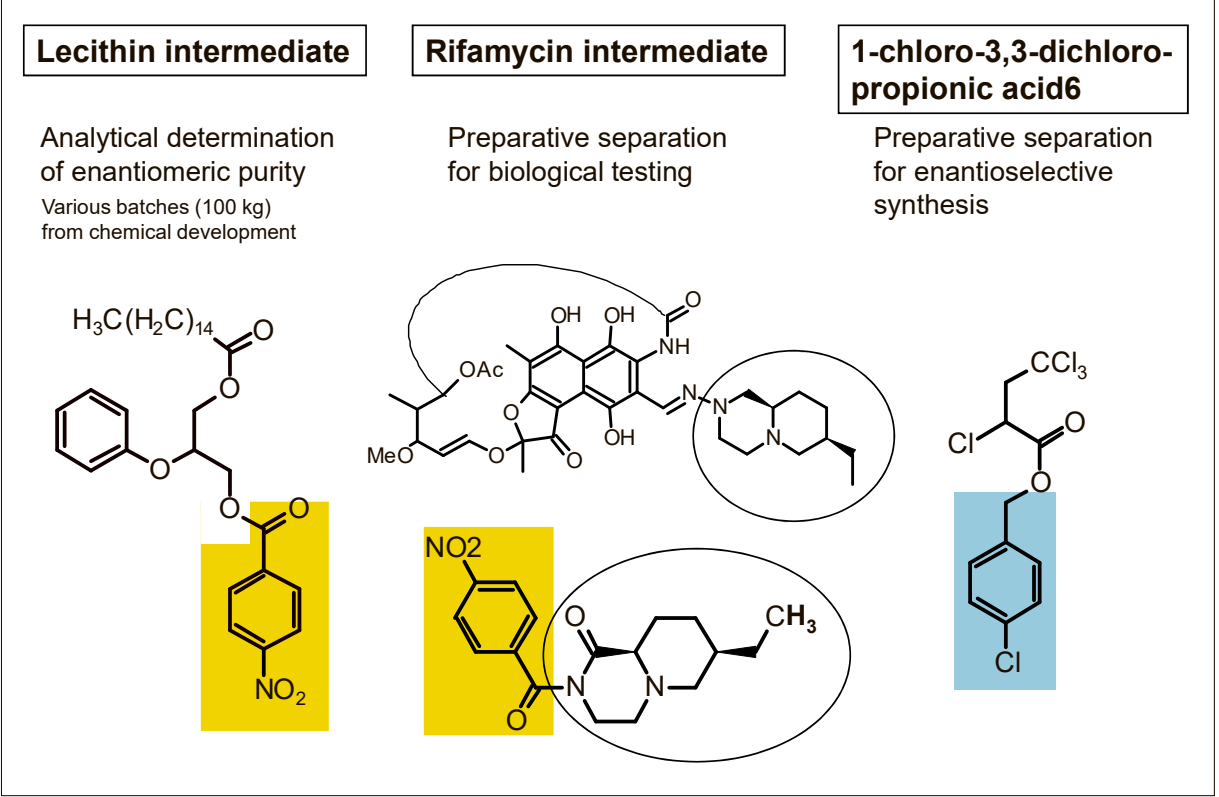

Fig. 5. Strategy of derivatization for enantioselective chromatography on CTA I. Left: Nitrobenzoyl ester of lecithin intermediate; Middle: Nitrobenzamide derivative of a synthetic intermediate for synthesis of Rifamycin analogous; Right: 4-chlorobenzyl-2,4,4,4-tetrachlorobutanoate. prevented the use of the 'classical' derivatization with a chiral agent to perform the pharmacokinetic study. The mild derivatization with diazomethane permitted the chiral integrity of the benzylic carbon atom to be maintained, enabling the enantioselective analysis (Fig. 6)

With the growing experience in the field, the separation of the enantiomers of an increasingly broad variety of racemic molecules could be achieved on CTA I. The applications included gamma- and delta-lactones for the preparation of antibiotics, ${ }^{[18,19]}$ chiral solvating agents for NMR, ${ }^{[20]}$ chiral titanocene, ${ }^{[21]}$ phenoxypyridyl and phenoxyphenyl biocide intermediates, ${ }^{[22-25]}$ organic conductors, and numerous drugs or drug intermediates..$^{[24-27]}$ The structure of selected chiral molecules of practical interest is shown in Fig. 7. It was also noticed that aromatic compounds having a chiral center in a benzylic position and carrying an unsaturated double bond $(\mathrm{C}=\mathrm{C}, \mathrm{C}=\mathrm{O}, \mathrm{C}=\mathrm{N}, \mathrm{C}=\mathrm{S})$ in $\alpha$ of this benzylic position were generally well resolved on CTA I.

Considering the high success rate of CTA I as a widely applicable CSP for enantioselective separations and the internal demand for increasing amounts of pure enantiomers on preparative scale, we decided in 1985 to build up a large CTA column (1 meter by $20 \mathrm{~cm}$ i.d.) containing about $14 \mathrm{~kg}$ of cellulose triacetate (CTA I) in the central research laboratories of former Ciba-Geigy (Fig. 8). Although the one-step synthesis of CTA I is relatively easy, the preparation of tens of kilograms required extended optimization of the reaction conditions. In particular the type of catalyst, temperature, and time were found to considerably affect the morphology of the material. Indeed, the sieving step to sort the broken materials according to particle size was a tedious operation but it was essential to eliminate small particles which cause the obstruction of the frits. Of course, the availability of the starting material cellulose in large amounts and the extremely high loading capacity, as well as the low production costs were other advantageous features of CTA I with respect to its utilization for large scale separations.

A number of practical applications to support the pharmaceutical and agrochemical divisions of Ciba were successfully achieved on this column. To our knowledge, this column was the largest 'chiral' column built at that time. This step was determining to persuade the chemists that enantioselective chromatography was a viable practical technique to rapidly access enantiomerically pure compounds (drug or drug intermediates) on a preparative scale. As a consequence, the number of requests for chromatographic separations started to rapidly grow from the mid-eighties. This 
was important not only in the context of testing the biological activity of the single stereoisomers but also in terms of intellectual property protection. Indeed, at that time and obviously before, many chiral drug molecules were still commercialized as racemates and it was common that the single enantiomers had not been separated. This situation left the door open for some companies such as Sepracor to prepare and patent the single enantiomers of marketed drugs, making a profitable business. ${ }^{[28]}$

Large-scale applications (hundreds of grams) on this column included for example the separations of enantiomers of the NSAI drug Oxindazac (benzylester), ${ }^{225]}$ $\mathrm{N}$-acetylindoline-2-carboxylic acid (precursor of an ACE inhibitor), ${ }^{[25]}$ the fungicide Clozylacon, ${ }^{[25]}$ 1-phenylethanol,[25] phenyldioxane,[25] Troeger's base, ${ }^{[25]}$ of the chiral solvating agent trifluoranthranylalcohol, ${ }^{[25]}$ the chiral reducing agent 1-tert-butylphenylethyl borane, ${ }^{[25]}$ and of ethylthiocyclohexene.[25] Fig. 9 and 10 show the chromatograms of the large-scale enantiomeric separation of selected chiral compounds on CTA I. For the fungicide Clozilacon (Fig. 10b), more than $1 \mathrm{~kg}$ of racemate was processed. ${ }^{[29]}$ At this time, the enantioselective separation of such amounts by chromatography was remarkably exceptional.

The successful application of CTA I motivated us to more deeply investigate the mechanism of the chiral discrimination between CTA I and chiral molecules, pursuing the ultimate goal of designing more efficient and possibly a universal CSP. In order to gain more insights into the interaction mechanism, a series of structurally related racemic compounds were synthesized and chromatographed on CTA I. ${ }^{[30]}$ The chromatographic data were correlated to selected properties of the chiral molecules, including the charge distribution on the van der Waals envelope and the molecular structure. It was found that both the electrostatic potential in the neighborhood of the chiral center (Fig. 11) and the flatness of the molecule were very important factors favoring a strong interaction with CTA I. In particular the interaction with the most retained enantiomer of delta-phenyl-valerolactone and the enantioselectivity of the enantiomeric separation were extremely high (Fig. 11a). The enantiomer showing the highest affinity for CTA I was retained 23 times more than the first eluting enantiomer. This molecule

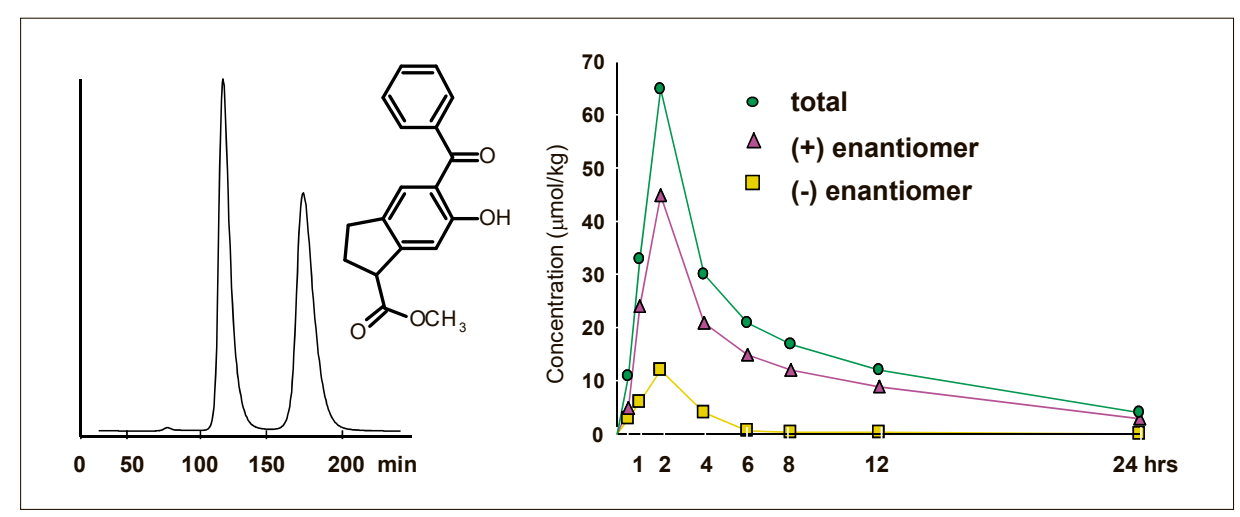

Fig. 6. a) Analytical separation of the enantiomers of oxindanac methyl ester; b) Stereoselective pharmacokinetic of oxindanac enantiomers.<smiles>Nc1ccc(C2C3CCC2C(=O)N3)cc1</smiles>

Antiaromatase<smiles>CC(=O)OC1c2ccccc2C(=O)N(C)c2ccccc21</smiles>

Anticonvulsive<smiles>CCCCC1(C)Cc2c(ccc3c2OC(C(=O)OCC)O3)C1=O</smiles>

Diuretic<smiles>CCN(C(=O)OCc1ccccc1)C(C)P(=O)(O)OC</smiles>

Antidepressive intermediate<smiles>CC(O)c1cccc(Oc2ccccc2)n1</smiles>

Pyrethroid insecticide intermediate<smiles>N#Cc1ccc(C2CCCc3cncn32)cc1</smiles>

Anticancer<smiles>CC[C@H]1Sc2sc(=S)sc2S[C@@H]1CC</smiles>

Organic conductor<smiles>C#CCOC(=O)C(C)Oc1ccc(Oc2ncc(Cl)cc2F)cc1</smiles>

Herbicide<smiles>COC(=O)C1(c2ccc(Cl)cc2Cl)CO1</smiles>

Herbicide intermediate<smiles>CC1COc2ccccc2N1C(=O)C(Cl)Cl</smiles>

Safener<smiles>CCn1cnc2c(Nc3cccc(Cl)c3)nc(NC3CCCCC3N)nc21</smiles><smiles>COC(=O)CC1CCC(=O)O1</smiles>

Antibiotic intermediate<smiles>CC(=O)C1Cc2ccccc2N1C(C)=O</smiles>

ACE-Inhibitor intermediate

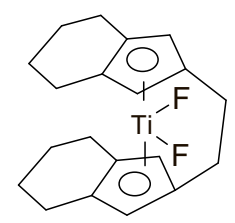

Chiral catalyst<smiles>CC(C)(C)c1ccccc1</smiles>

Chiral reducing agent<smiles>CC(C)(C)N1CC(=O)OC1C(=O)OCc1ccccc1</smiles>

Chiral synthon 
Fig. 8. Picture of large CTA I column (Central Research Laboratories; CibaGeigy 1985); column $(20 \mathrm{~cm} \times 100 \mathrm{~cm})$ packed with $14 \mathrm{~kg}$ CTA I.

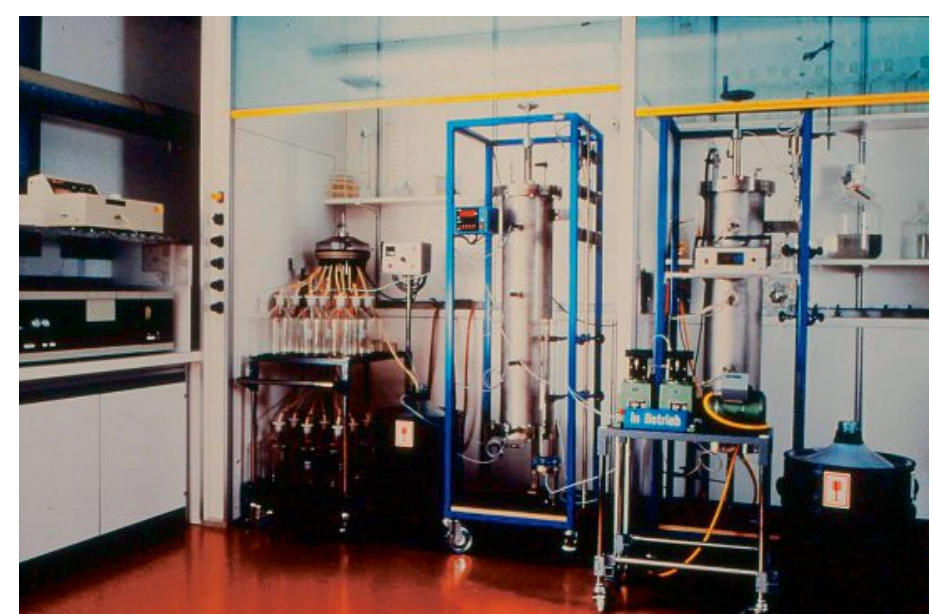

Fig. 9. Applications of large scale enantiomeric separations on CTA I (large column).

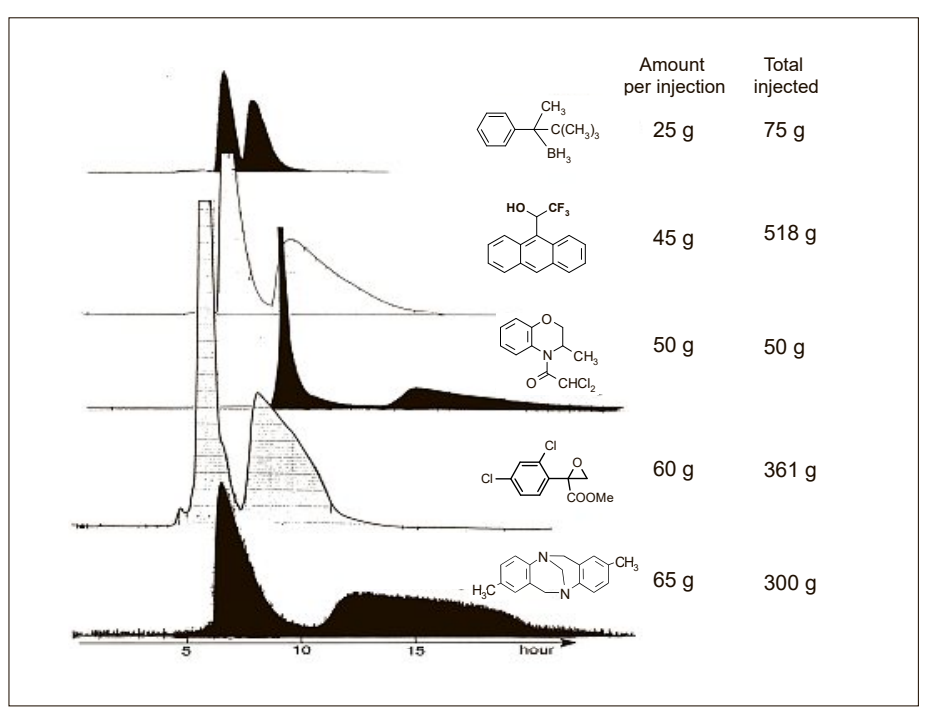

a

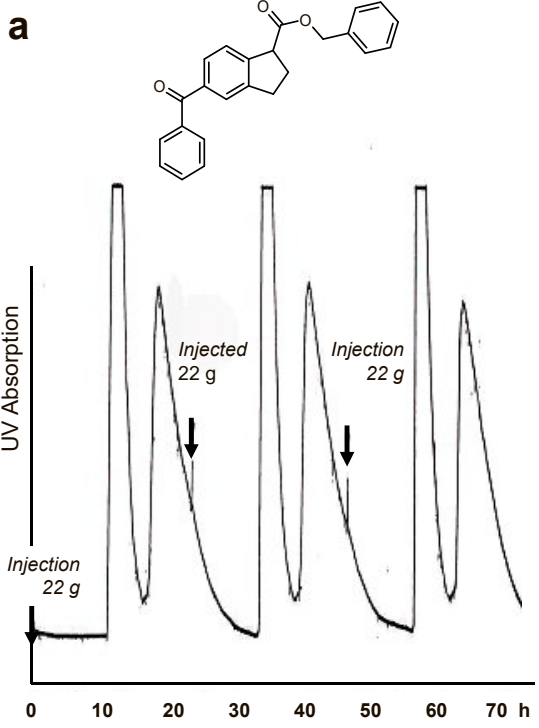

b
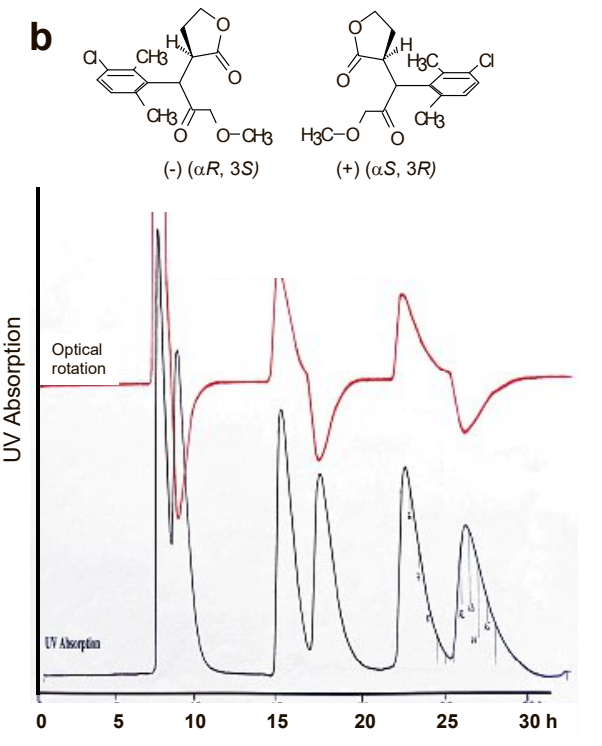

Fig. 10. Preparative chromatographic resolution on cellulose triacetate I (large column $20 \mathrm{~cm}$ $x 100 \mathrm{~cm}$ ) of a) racemic oxindanac benzylester. Stacked injections (each $22 \mathrm{~g}$ ); mobile phase, ethanol-water 94:6; flow rate: 5 litre/h pressure, 1.6 bar; b) racemic clozalicon; mobile phase, ethanol-water 94:6; flow rate: 5 liter/h; pressure: 1.6 bar, Injection, $52 \mathrm{~g}$; run time, $30 \mathrm{~h}$ including 2 recycling. was later used as a model to 'titrate' the interaction of the individual enantiomers with CTA I. ${ }^{[31]}$

Although the results of this theoretical studies were inspiring, it rapidly became clear that such a conclusion was only valid for the studied class of structure and that the complex molecular arrangement of CTA I with the presumed presence of many different interaction sites make this approach very uncertain for predicting the enantioselectivity of a separation.

\section{Polysaccharide Beads CSPs}

Because not all racemic molecules could be separated on CTA I, we continued, in parallel to the mechanistic investigations and practical applications, to evaluate the potential of further polysaccharide derivatives as chiral packing material for chromatographic resolutions. Considering that our main focus was on the preparative application of enantioselective chromatography to support the life science departments, we developed a process to prepare pure polysaccharide derivatives and shaping them in a form which was appropriate for packing a column. ${ }^{[32-34]}$ In the meantime, another process was developed in Japan by Okamoto and his group to produce polysaccharide-based stationary phases. ${ }^{[35-37]}$ However, this process which consists in coating the polysaccharide derivative on silica gel (about 20/80 w/w), considerably reduces the content of 'chiral polysaccharide selector' in the packing material, and consequently the loading capacity of the stationary phase.

Our process to prepare pure polysaccharide derivatives consisted in producing an aqueous emulsion of the polysaccharide derivatives dissolved in a mixture of an organic solvent and a precipitation agent, in the presence of a surfactant (Fig. 12). By slowly heating the emulsion, the organic solvent contained in the droplets was gradually removed, leaving the solid polysaccharide derivative as small beads which could be easily filtrated. ${ }^{[32-34]}$ The influence of various parameters on the size of the beads, their size distribution, and the specific surface area of the produced material was investigated in detail. Parameters such as the type of surfactant, the type of organic solvent, the type of precipitation agent, the speed of rotation of the stirrer, the temperature, and the heating time were varied.

For benzoylester derivatives of cellulose, sodium lauryl sulfate was found to be the best surfactant for the emulsion while for arylcarabamate derivatives, polyvinylalcohol was more effective. The best conditions had to be optimized for each polysaccharide derivative. It was noticed that the chiral recognition ability was dependent not only on the crystallinity, like for CTA I, but also very much on the specific surface 
area of the obtained material. For benzoylcellulose for example, a domain comprising a $\Delta \mathrm{H}$ in differential scan calorimetry (crystallinity) ranging between 15 and 23 $\mathrm{J} / \mathrm{g}$ and a specific area ranging between 40 and $70 \mathrm{~m}^{2} / \mathrm{g}$ was found to be optimal (Fig. 13). The particle size of the beads was mostly controlled by the speed of rotation of the stirrer, but in some instances, sieving the material was necessary in order to narrow the particle size distribution, which is important to obtain a regular packing and high performance column.

A variety of pure polysaccharide-based beads CSPs were prepared according to this process (Table 2), and a few typical pictures of the obtained beads (electron microscopy) are shown in Fig. 12.

Excellent analytical separations were obtained on the pure polysaccharidebased phase. ${ }^{[33,38,39]}$ In contrast to CTA I, racemic benzylic alcohols were easily directly resolved in the underivatized form on tribenzoylcellulose beads (TBCB). ${ }^{[33]} \mathrm{A}$ broad variety of structurally diverse chiral molecules were actually well resolved on TBCB. Published examples include a series of pyridyl pyrethroid intermediates, ${ }^{[20]}$ chiral lactones, ${ }^{[38]}$ diols (as acetates), ${ }^{[33,38]}$ epoxides, ${ }^{[38]}$ chiral sulfoxides, ${ }^{[33]}$ chiral mono- and dihydropyranes, ${ }^{[33]}$ chiral indane derivatives, [38] barbiturates, ${ }^{[38]}$ hypnotics, ${ }^{[38]}$ atrope isomers, ${ }^{[38]}$ and various other chiral drugs. ${ }^{[26,38]}$

However, above all it is the preparative potential of TBCB as a chiral chromatographic material which is remarkable. Indeed due to the high density of chiral selector in TBCB, the material exhibits a much higher loading capacity compared to the same type of chiral stationary phase prepared by coating on a silica gel carrier (Chiralcel OB). This advantage has been exploited to solve several practical applications for which larger amount of pure enantiomers were needed. Examples of application to chiral substances developed at Ciba-Geigy are shown in Figs 14 and 15. Fig. 14 shows the application to the key chiral intermediate for the anticancer agent 'Edatrexate'.[26,27,39] Fig. 15 shows the separation of the key chiral intermediate in the synthesis of the insecticide 'Lufenuron' which has become one of the most important insecticides against fleas in dogs and cats (Fig. 15b) ${ }^{[24,40]}$ and of a formerly nootropic development drug (Fig. 15a). ${ }^{[25-27]}$ Further preparative applications include the anti-cancer drug Fadrozole, ${ }^{[41]}$ a synthetic protein intermediate, ${ }^{[42]}$ and herbicide and insecticide intermediates. ${ }^{[20]}$

Other pure polysaccharide-based phases (beads) were synthesized and applied. ${ }^{[24,38]}$ Interestingly, we found that, even though the structural changes are far away from the chiral basic glucose element of the polysaccharide chain, the

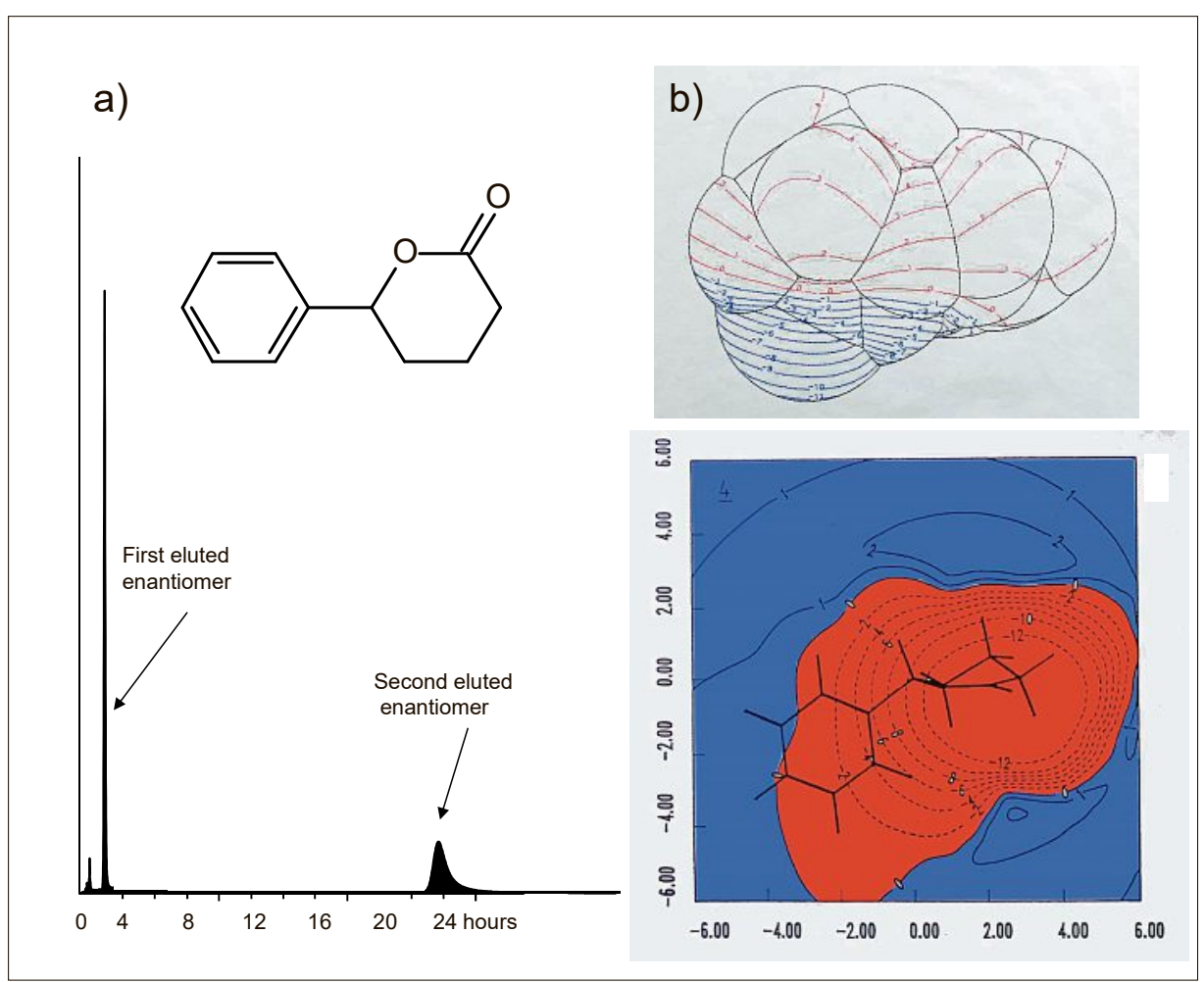

Fig. 11. Delta-phenyl valerolactone. a) Structure and chromatographic separation of the enantiomers; b) electrostatic potential contour (tridimensional and map). Contour lines are given in $\mathrm{kcal} \mathrm{mol}^{-1}$ (negative region is in red, positive in blue).

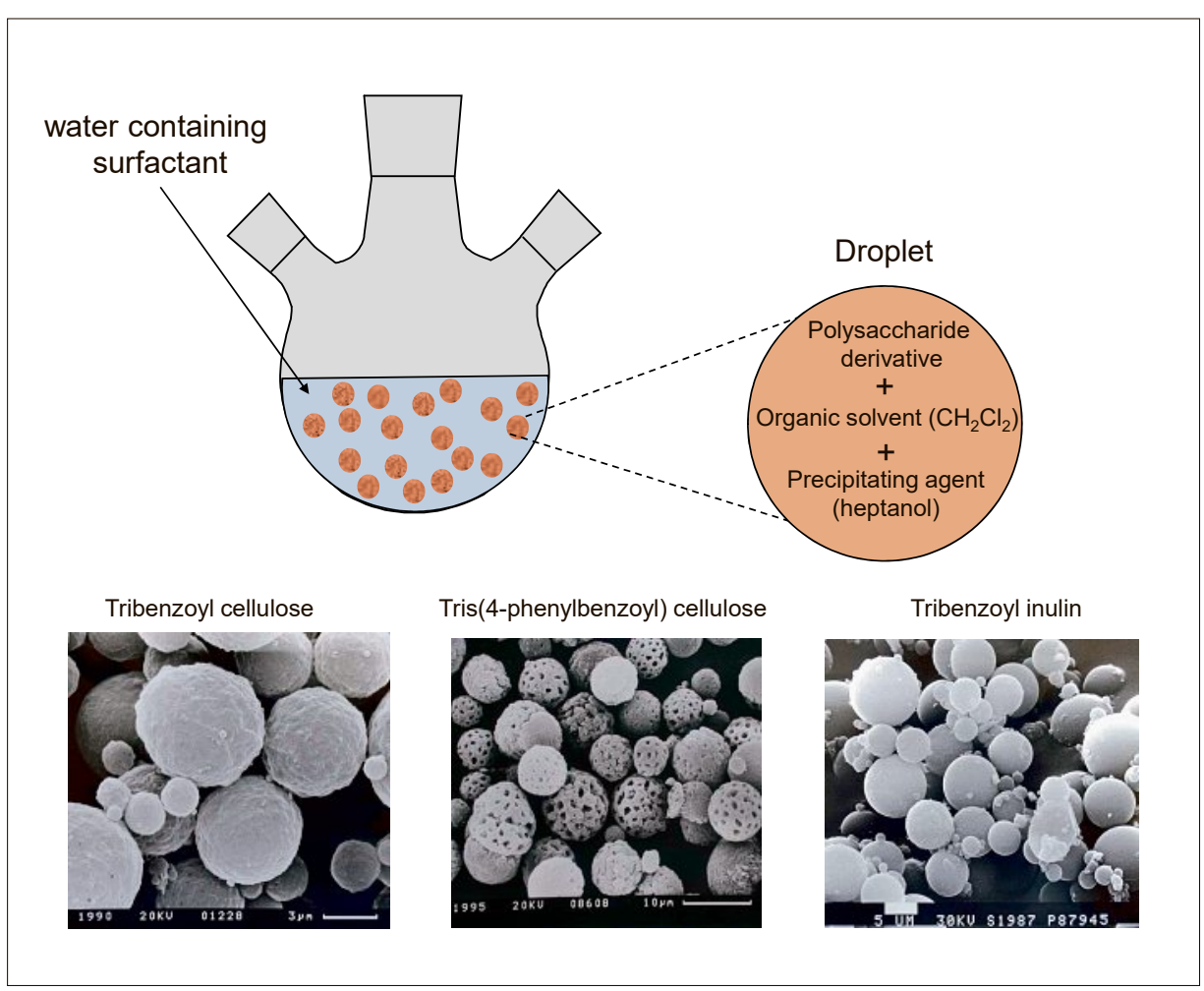

Fig. 12. Process of preparation of beads of polysaccharide derivatives.

CSPs beads made from the three ortho-, meta, and para-methyl benzoyl cellulose derivatives behave differently in terms of chiral recognition ability. Some chiral molecules are resolved of only one of the three phases, and in a few instances there is even inversion of elution order of the respective enantiomers under identical chromatographic conditions as illustrated in Fig. 16. ${ }^{[38]}$ This observation again points to the importance of the supramolecular structure of the CSP in the chiral recognition process. Very likely, change of the position of the methyl group on the benzoyl moiety leads to important alterations of the shape of the chiral receptors 
due to modification of the supramolecular arrangement of the polymer chains and/or by the actual conformation of the benzoyl cellulose chains. Although achiral per se, the methylbenzoyl groups can obviously transfer the chiral information of the sugar moiety to which they are attached, further into the space around the polymer chains, creating different chiral cavities.

The variable selectivity of the methylbenzoylcellulose-based CSPs provided a useful tool to improve the chromatographic resolution of racemic compounds. Many chiral substances were resolved on a preparative scale on these phases and include external collaborations. ${ }^{[42,43]}$ Unique enantioselectivity could be achieved for several important drug and biocide candidates on the methylbenzoyl cellulose beads phases. Applications include the enantiomers of anti-cancer drug glutethimide, ${ }^{[38]}$ the enantiomers of the chiral key intermediate for the synthesis of a new class of cyclopentane-type fungicides, ${ }^{[44]}$ and the interesting separation of the enantiomers of a chiral aziridine and oxaziridine containing a chiral center on the nitrogen atom ${ }^{[38]}$ (Fig. 17).

Like for CTA I, the achiral derivatization strategy was also applied to improve chromatographic resolution of racemic alcohols on beads of substituted-benzoylcellulose derivatives. ${ }^{[45]}$ Derivatization of racemic alcohols to their corresponding benzoate esters was generally very beneficial in terms of improving enantioselectivity, in particular on the tribenzoylcellulose CSP. However, the nature and position of the substituent on the derivatizing benzoyl group was clearly critical for the separation outcome as shown in Fig 18 for the three methoxybenzoate derivatives of three different alcohols.

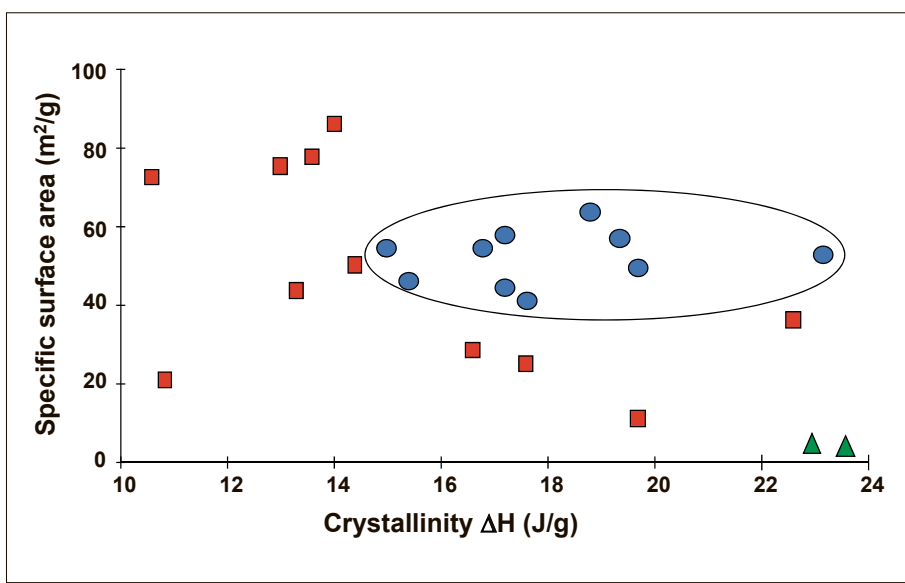

Fig. 13. Crystallinity and surface specific area of samples of tribenzoylcellulose beads. The circle represents the domain of samples exhibiting a good enantioselectivity and resolution.

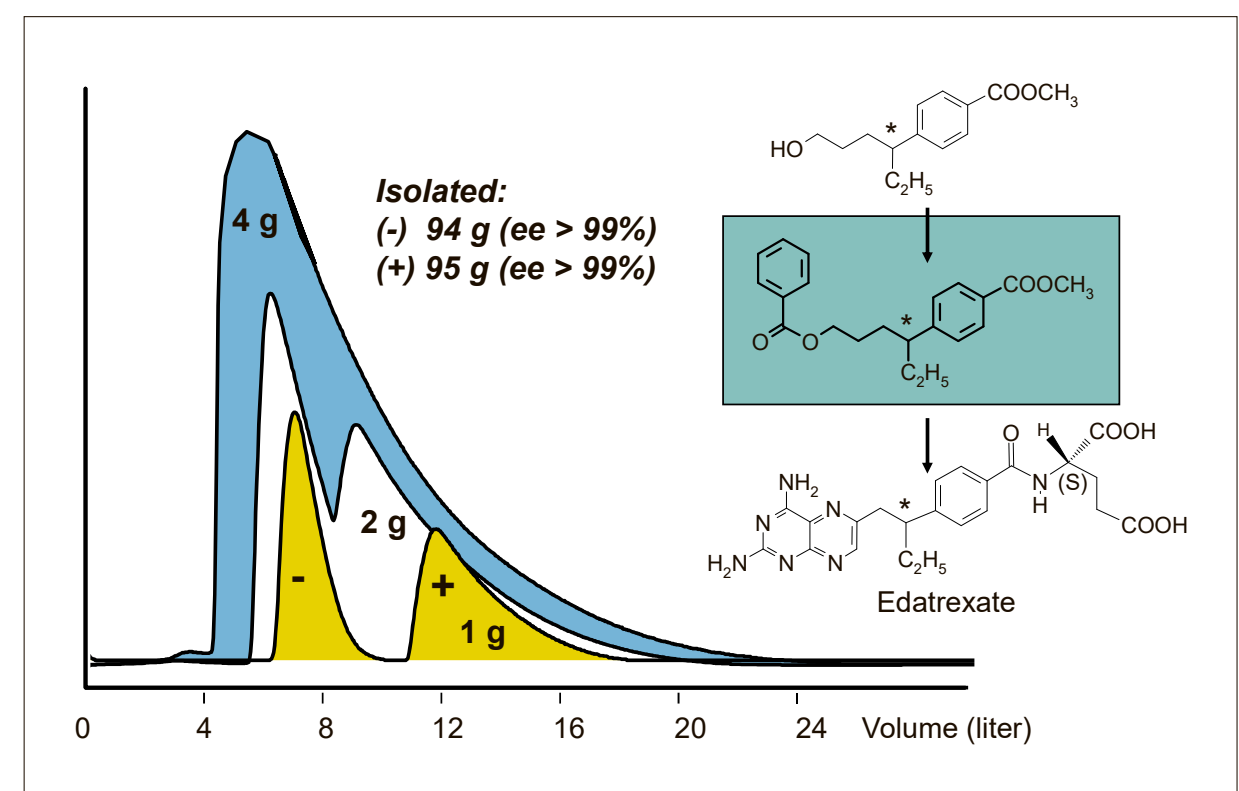

Fig. 14. Preparative chromatographic separation of the enantiomers of the key intermediate for the synthesis of the anticancer drug edatrexate on tribenzoylcellulose beads. Column, $5 \mathrm{~cm}$ x $70 \mathrm{~cm}$; mobile phase hexane/2-butanol 9/1; Flow rate, $60 \mathrm{ml} / \mathrm{min}$

Table 2. Name of the prepared polysaccharide-based bead stationary phases

\begin{tabular}{|l|l|}
\hline Name & Name \\
\hline Tribenzoyl cellulose & Tris-(4-butylbenzoyl) cellulose \\
\hline Tris-(2-methylbenzoyl) cellulose & Tris-(4-phenylbenzoyl) cellulose \\
\hline Tris-(3-methylbenzoyl) cellulose & Tris-(4-tert-butylbenzoyl) cellulose \\
\hline Tris-(4-methylbenzoyl) cellulose & Tris-(naphtoyl) cellulose \\
\hline Tris-cinnamoyl cellulose & Cellulose tris-(3,5-dimethylphenylcarbamate) \\
\hline Tris-(4-ethylbenzoyl) cellulose & Cellulose tris-phenylcarbamate \\
\hline Tris-(4-chlorobenzoyl) cellulose & Tricrotonyl cellulose \\
\hline Tris-(3-chlorobenzoyl) cellulose & Tribenzoyl amylose \\
\hline Tris-(4-fluorobenzoyl) cellulose & Tris-(2-methylbenzoyl) amylose \\
\hline Tris-(3,5-dichlorobenzoyl) cellulose & Benzoylguaran \\
\hline Tris-(3,4,5-trichlorobenzoyl) cellulose & Tribenzoyl inulin \\
\hline
\end{tabular}

Tris-(dibromobutyryl) cellulose 

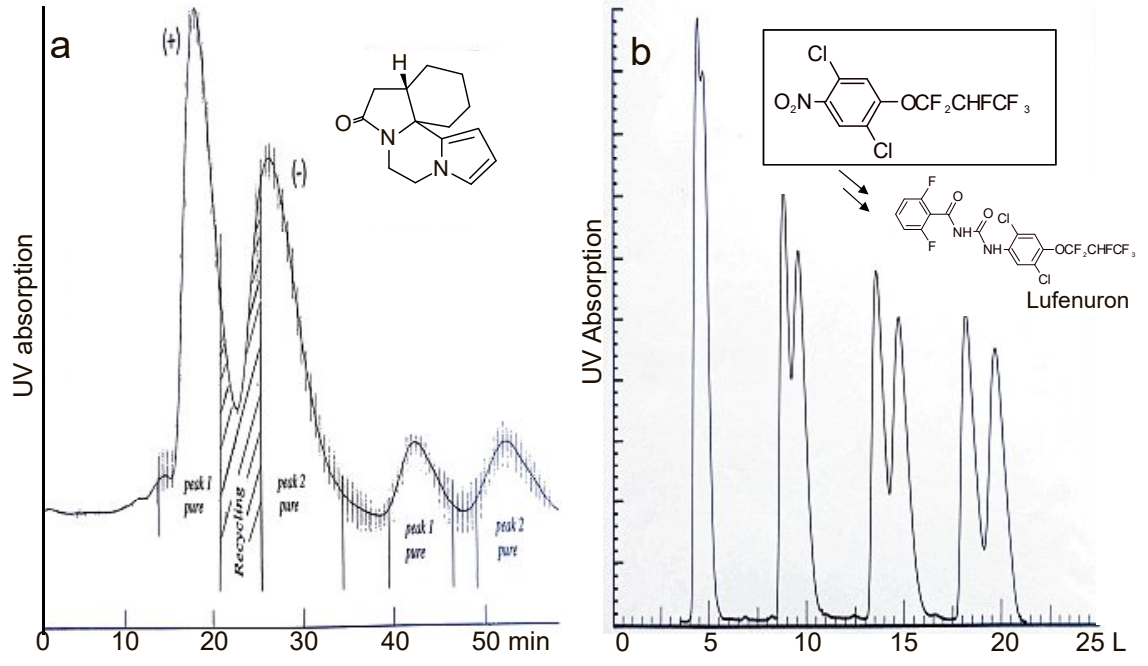

Fig. 15. Preparative chromatographic separations on tribenzoylcellulose beads a) enantiomers of a tetracyclic nootropic drug. Column, $5 \mathrm{~cm} \times 45 \mathrm{~cm}$; mobile phase, methanol 100\%; flow rate, $60 \mathrm{ml} / \mathrm{min}$; b) enantiomers of Lufenuron intermediate. Column: 5 x $70 \mathrm{~cm}$; Injection: 1-4 g; mobile phase, hexane/2-butanol 9:1; flow rate, $60 \mathrm{ml} / \mathrm{min}$.

This spectacular effect shows again that the strategy of achiral derivatization can be very useful for separation on polysaccharide-based phases, especially for preparative applications and permits to extend the applicability of a specific CSP. However, the question regarding how to predict what is the best derivative for a defined CSP remains.

Beads made from the pure 3,5-dimethylphenylcarbamate of cellulose (DMPCCbeads) were also prepared.[34] This chiral selector is commonly applied in the silicagel-coated form. ${ }^{[36]}$ The emulsion process is similar to the process applied to the ben- zoyl derivative of cellulose or amylose, but polyvinyl alcohol was found to be a more effective surfactant whereas N-phenyl-1heptylcarbamate gave the best results as the precipitating agent in terms of porosity of the beads material.

Again in this case, the pure polysaccharide material exhibits a much higher loading capacity compared to the silica-coated phase. From the determinations of the peak saturation for the pure DMPCC-beads (Fig. 19a) and the coated 3,5-dimethylphenylcarbamate of cellulose column (Fig. 19b), it has been estimated that the pure phase has a loading capacity

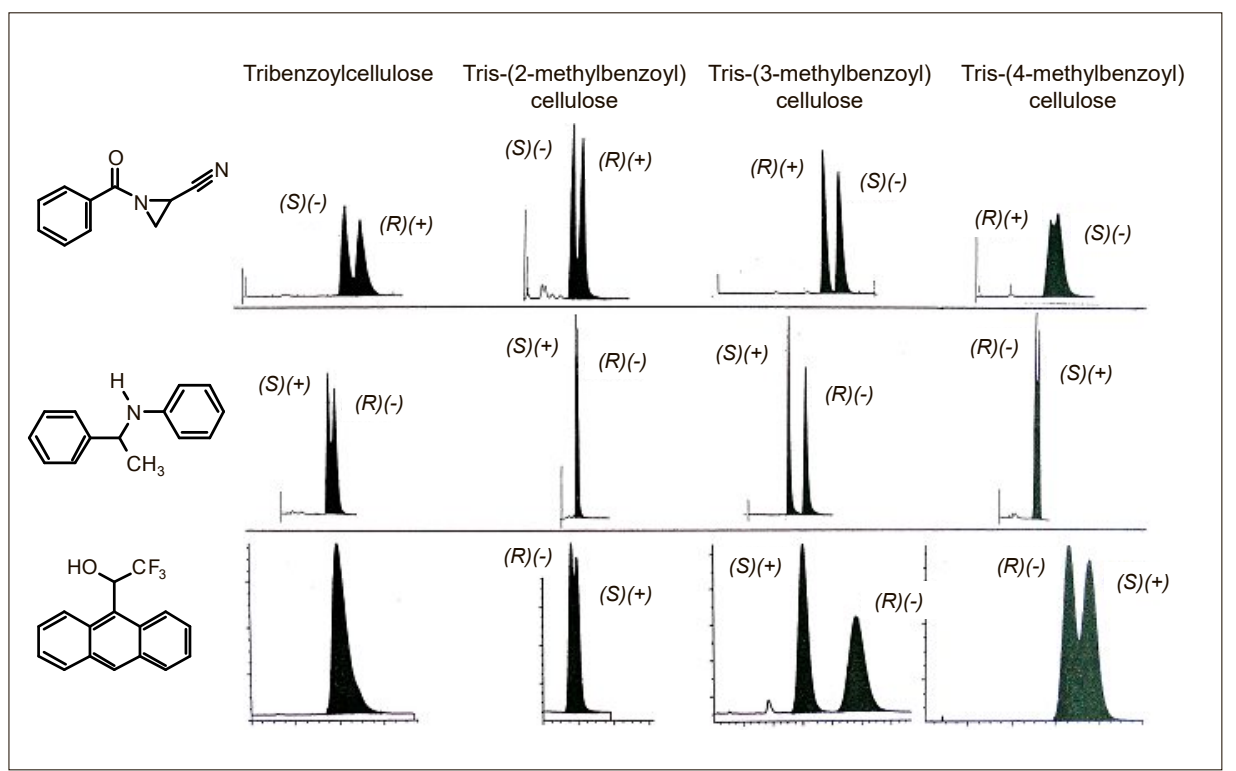

Fig. 16. Analytical separations of the enantiomers of selected compounds on ortho-, meta-, and paramethylbenzoyl cellulose beads. Influence of the position of the methyl substituent on the phenyl ring on chiral recognition ability. Column, $0.46 \mathrm{~cm} \times 25 \mathrm{~cm}$; mobile phase, hexane/ 2 propanol 9/1; flow rate, $1 \mathrm{ml} / \mathrm{min}$. which is almost 20 times higher in $\mathrm{mg} / \mathrm{g}$ of phase and 5 times higher in $\mathrm{mg} / \mathrm{ml}$ of phase for the pure cellulose derivative. The peak saturation was determined by injection of increasing amounts of racemate on the respective columns.

Although CTA I and the polysaccharide-based bead materials were quite successfully applied for numerous practical enantioselective separations, their utilization needed some caution because these materials have limited mechanical stability, preventing them to be operated at elevated pressure. High pressures caused breakage of the particles, leading to an irreversible compression of the chromatographic bed and clogging of the column. This feature was also limiting in terms of throughput because only moderate flow rate could be applied. For this reason, the utilization of CTA I and polysaccharide-based beads has gradually decreased to the benefit of mechanically more stable chromatographic materials.

As mentioned above, another technique of preparation of polysaccharidebased phase was developed by the group of Okamoto in Japan in the early eighties ${ }^{[35-37]}$ and a number of columns packed with these materials were commercialized from 1984. In this process, the polysaccharide derivatives (about $20 \mathrm{wt} \%$ ) are physically absorbed by coating on the surface of silica gel $(80 \%)$, conferring mechanical stability to the chromatographic material.

\section{Immobilized Polysaccharide-based CSPs}

The silica-gel-coated phases developed by Okamoto and his group were definitely more effective in terms of chromatographic properties. Compared to the pure polysaccharide-based phases, the silica-coated phases exhibit a much lower loading capacity due to the presence of about $80 \%$ of achiral supporting carrier (silica gel), but their higher mechanical robustness permits operation at high pressure, resulting in higher flow rates and productivity.

However, the coated phases showed another major limitation due to their high solubility in most organic solvents. This restriction prevents the utilization of such solvents for chromatographic separations. While this constraint is less important for analytical applications, it is critical for preparative purpose. Indeed, for preparative applications, productivity is a major parameter and it is, among other factors, largely related to the solubility of the solute in the mobile phase. It is established that many racemic compounds are poorly soluble in the solvents which are compatible with the coated polysaccharide-based CSPs. Moreover, unlike other types of CSPs, the mobile phase type and compo- 
sition greatly affect the chiral discrimination process with polysaccharide-based CSPs, thus considerably influencing the enantioselectivity of the chromatographic separations. A few examples illustrating the solvent effect on enantioselectivity are shown in Fig. 20.

In order to tackle this problem we designed and developed a new concept to immobilize the polysaccharide derivatives on the carrier material. ${ }^{[46-52]}$ The general process flow for immobilization on silica gel is shown in Fig. 21.

The process consists of a photochemical or thermal treatment of the silica gel carrier after coating of the polysaccharide derivatives (Fig. 22). In the photochemical approach, the silica-gel-coated material is suspended and stirred in an inert solvent and this suspension is irradiated for a few hours using an immersed highpressure mercury lamp (Fig. 22a). The suspension solvent and irradiation time were found to influence the degree of immobilization and the enantioselectivity of the chromatographic material. In the thermal process, the suspension is heated in the presence of a radical initiator such as AIBN or tert-butyl peroxide (Fig. 22b). Type and amount of radical initiator, and solvent suspension, have a critical influence on the degree of immobilization and enantioselectivity of the obtained chromatographic material.

The processes are generally applicable but the optimal conditions are very dependent on the substituents present on the polysaccharide backbone, i.e. for each polysaccharide derivative hundreds of experiments had to be performed. For some polysaccharide derivatives the photochemical process was more effective while for others the thermal process was more valuable (Table 3). It was generally found that prolonging the irradiation time more than 20 hours does not further increase the immobilization degree. ${ }^{[52]}$ For a few polysaccharide derivatives, addition of a photosensitizer such as thioxanthone was necessary to reach a reasonable immobilization degree. Table 3 shows the preferred immobilization process for a series of polysaccharide derivatives.

Although many physical and chemical investigations were performed to understand the mechanism of immobilization, no conclusive proof could be established to elucidate the immobilization process. However, as both processes imply the formation of radicals, it is reasonable to believe that the immobilization occurs through cross-linking between the polysaccharide chains and/or with silica gel, leading to the formation of an insoluble tridimensional network (Fig. 23). After the immobilization treatment, the material is extracted/washed to remove the non-

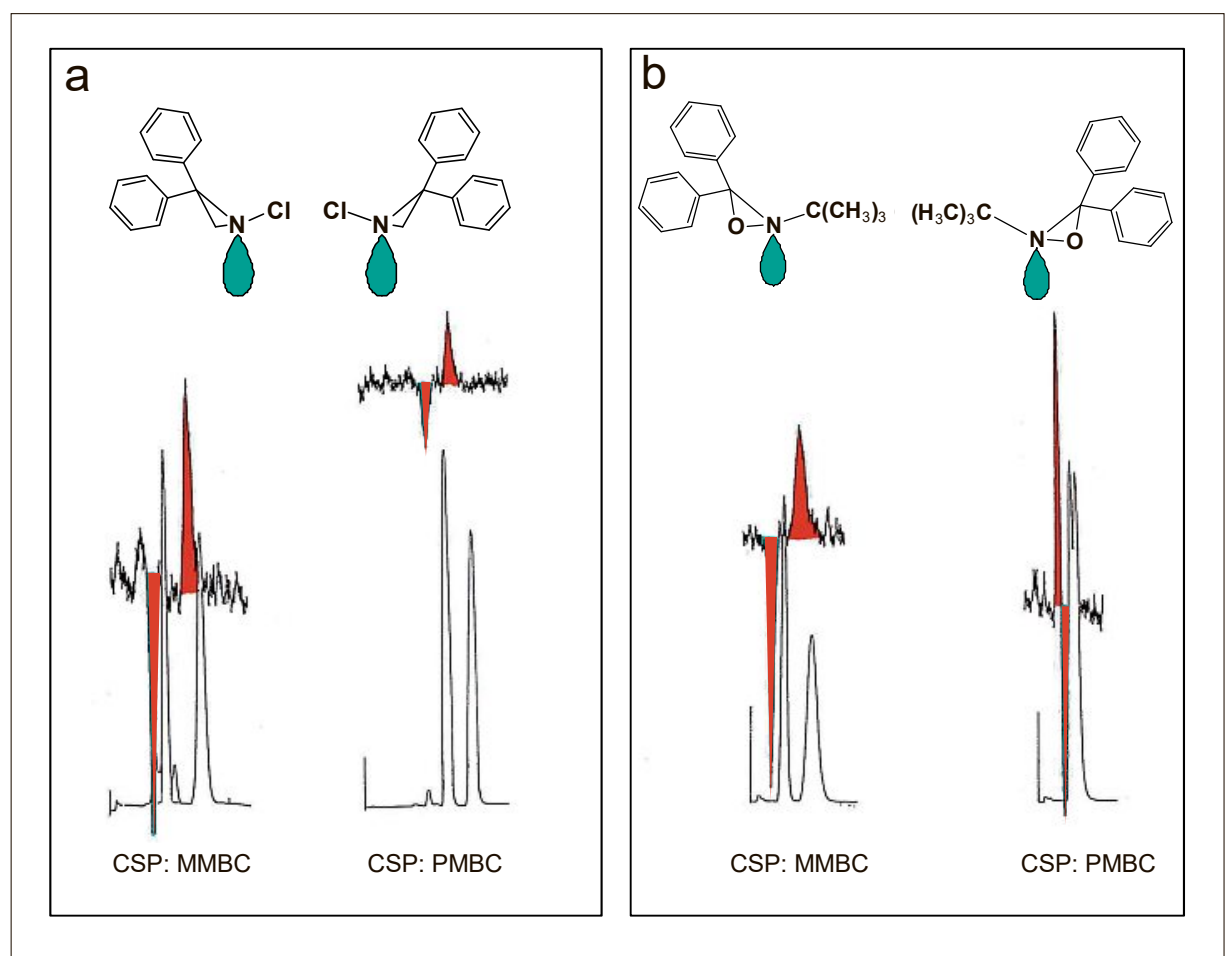

Fig. 17. Analytical separations of the enantiomers of compounds containing a chiral nitrogen atom. a) $\mathrm{N}$-chloro-diphenylaziridine; b) N-tert-butyl-diphenylloxaziridine. Column $(0.46 \mathrm{~cm} \times 25 \mathrm{~cm})$ packed with 3-methylbenzoylcelullose beads (MMBC) and 4-methylbenzoylcelullose beads (PMBC); mobile phase, hexane/2-propanol 9/1; flow rate, $1 \mathrm{ml} / \mathrm{min}$. Top line, optical rotation.

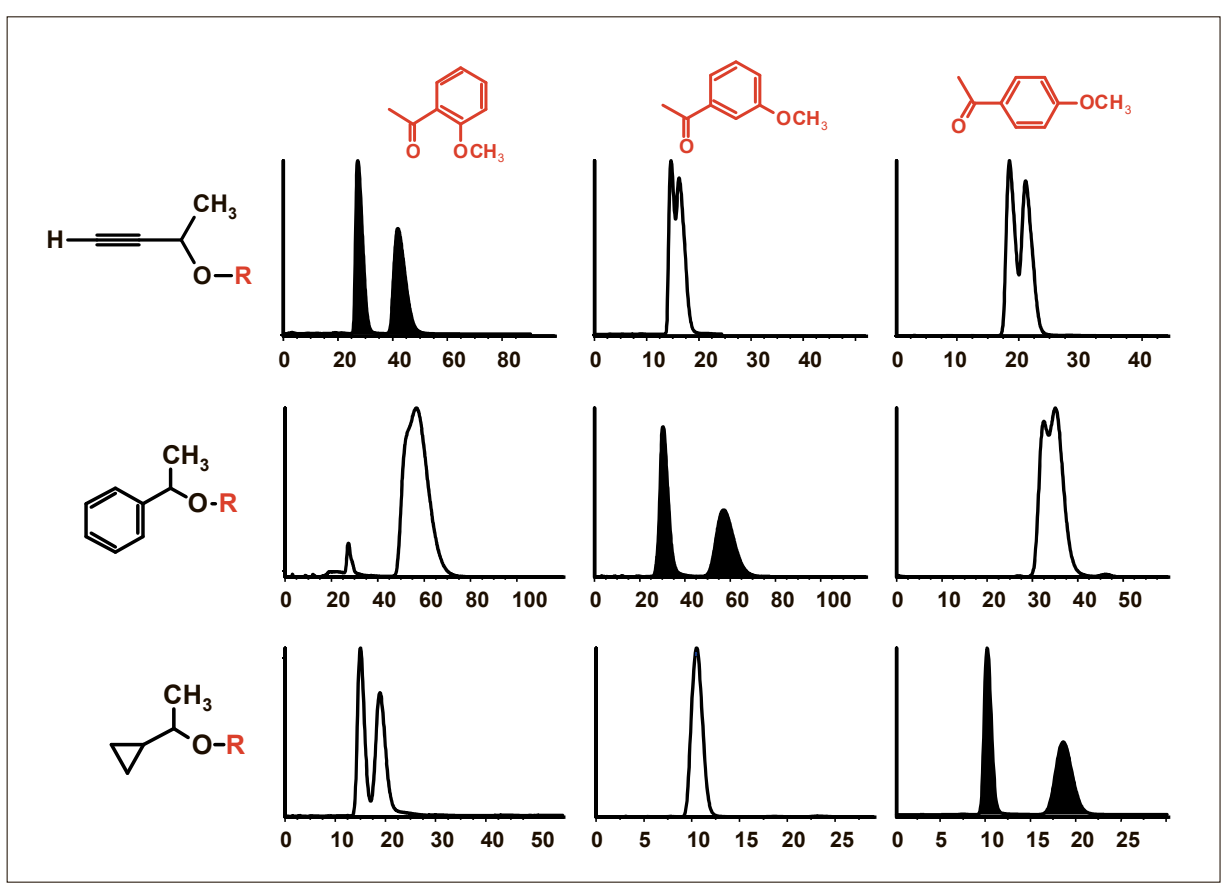

Fig. 18. Separation of the enantiomers of methoxybenzoylester derivatives of selected alcohols on tribenzoylcellulose beads. Column, $0.46 \mathrm{~cm} \times 25 \mathrm{~cm}$; mobile phase, hexane/2-propanol 9/1; flow rate, $1 \mathrm{ml} / \mathrm{min}$.

immobilized parts of the polysaccharide derivatives.

The immobilized phases show chiral recognition abilities which are similar to those obtained with the non-immobilized ones when applied under the same chromatographic conditions. There is usually a little price to pay in terms of enantioselectivity for the immobilized phases, i.e. small decrease of the separation performance, but this can be very often easily counterweighed by modifying the mobile phase composition. The possibility to use any kind of solvent with the immobilized phases has considerably enlarged the number of options to optimize enantioselective separations. Fig. 24 shows an example of resolution of a racemic compound on dif- 


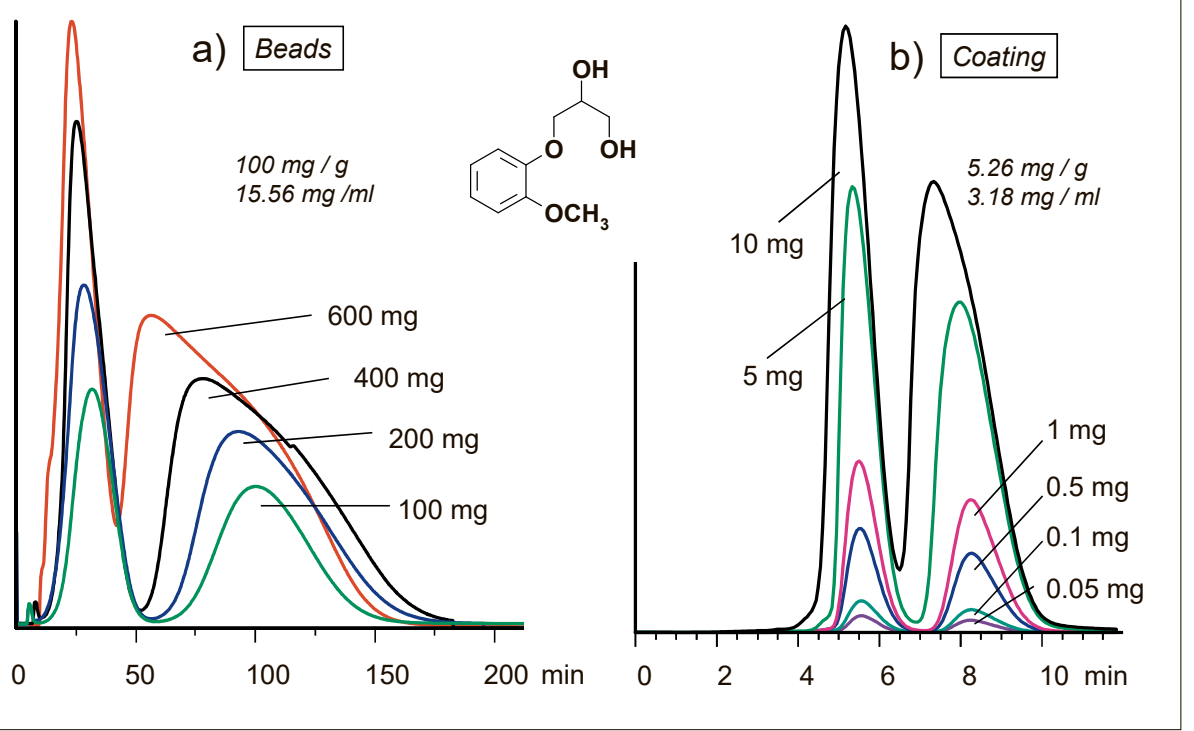

Fig. 19. Separation of the enantiomers of guaifenesine. a) on cellulose 3,5-dimethylphenylcarbamate beads. Column: $1.25 \mathrm{~cm} \times 26.6 \mathrm{~cm}$ (32.6 ml), packed with $5.4 \mathrm{~g} \mathrm{CSP}$; mobile phase: heptane/2-propanol 90/10; flow rate, $5 \mathrm{ml} / \mathrm{min}$. b) on silica gel coated cellulose 3,5-dimethylphenylcarbamate. Column: $0.4 \mathrm{~cm} \times 25 \mathrm{~cm}(3.14 \mathrm{ml})$, packed with $1.9 \mathrm{~g}$ CSP; mobile phase, heptane/ethanol 65/35; flow rate, $0.7 \mathrm{ml} / \mathrm{min}$.

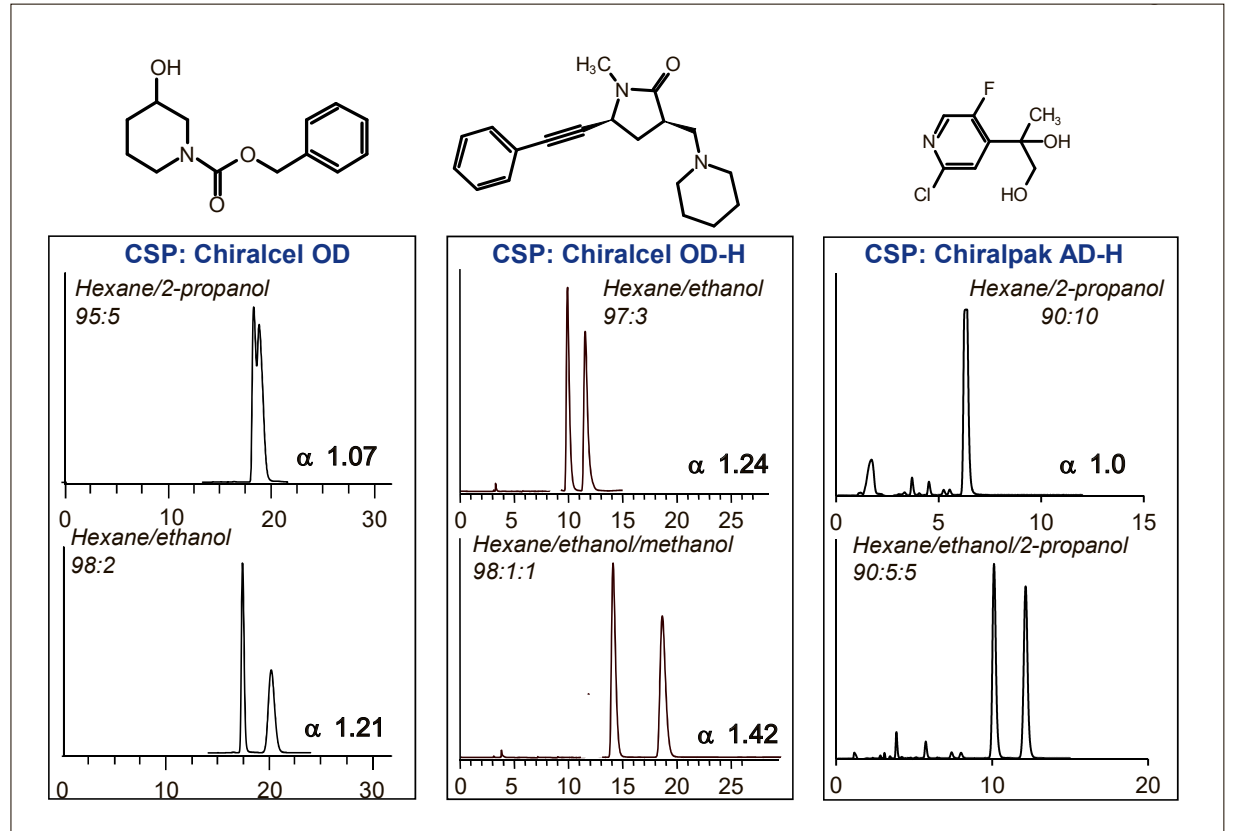

Fig. 20. Influence of mobile phase composition on enantioselectivity. Chromatographic separation of the enantiomers of three selected racemic compounds on coated polysaccharide-based stationary phases.

ferent immobilized polysaccharide-based phases and under different mobile phase conditions which would not be tolerated by the corresponding non-immobilized phases, demonstrating the extended versatility of the immobilized polysaccharide-based phases.

Examples illustrating the spectacular improvement of enantioselectivity are shown in Fig 25.

The processes of immobilization have been patented and in 1999, Daicel Chemical Industries, the leader in the field of chiral stationary phases, acquired the ted to solve numerous separation problems which could not be solved or only unsatisfactorily with the non-immobilized phases. The CSPs were particularly invaluable to resolve racemic compounds which were non- or poorly soluble in the chromatographic mobile phases which are tolerated by the conventional CSPs. The improved solubility of the chiral solutes to be separated offer also a considerable advantage in terms of productivity and competitiveness for large scale separations.

The main advantages of the immobilized polysaccharide base phase can be summarized as follows:

- Utilization of almost any kind of organic solvent as the mobile phase

- Possibility to extend the choice of polysaccharide-based CSPs to polysaccharide derivatives which are soluble in the 'classical' mobile phase

- Extended possibility to improve selectivity by modulating the mobile phase type and composition

- Extended possibility to modulate retention time according to the requirements (fast analytic, shorter cycle time in preparative applications)

- Possibility to solubilize or improve solubility of the solute in the mobile phase

- More stable columns possessing enhanced robustness (increased life time)

Our process is very simple and minimizes the possible disturbance of the conformation of the polysaccharide chains and of the supramolecular structure which are known to dramatically affect the chiral recognition ability. Since the discovery of our immobilization technique in 1994, a few other approaches for the preparation of immobilized polysaccharide-base phases have been developed but they all involve the introduction of chemical groups which disrupt the homogeneity of the substitution along the polysaccharide chain, and additionally need the elaboration of multi-step synthetic pathways, mostly requiring protection and de-protection steps. The methods of immobilization of polysaccharide derivatives have recently been reviewed. ${ }^{[53]}$

The immobilized chiral stationary phase prepared from 3,5-dichlorophenylcarbamate of cellulose is a remarkable example of a successful material that could not be applied in the non-immobilized form due to the solubility of the cellulose derivative even in apolar organic solvents. ${ }^{[50,54]}$ This immobilized CSP exhibits an exceptional recognition ability toward a broad variety of chiral substances and has been very much used in our laboratories at Novartis since 1999. It has been introduced on the market by Daicel in 2007. The versatility and usefulness of this phase has been demonstrated internally by hundreds of practical applications and the two examples below should illustrate its capability. The 
example in Fig. 26 shows the separation of the enantiomers of a chiral knot whose chirality is only due to the spatial arrangement of the molecule. ${ }^{[55]}$ This was the first example of separation of this kind of molecular chirality. The major problem was the insolubility of the molecule in the organic solvents which are tolerated by the nonimmobilized phase. With the immobilized phase, mixtures containing chlorinated solvents, could be applied, permitting the resolution of the enantiomers.

The impact of the application shown in Fig. 27 was extremely high as it has permitted to elucidate the mechanism of action of the immunosuppressant drug FTY720, ${ }^{[56,57]}$ which has recently been introduced on the market. In the context of the mechanistic investigations, the pure enantiomers of the mono-phosphate aminoalcohol were needed. Due to the high polarity of the phosphate molecule, attempts to separate the enantiomers directly failed. After double derivatization of the molecule (cyclic phosphate ester and oxazolidinone), preparative chiral resolution of the poorly soluble derivative could be achieved using the home-made immobilized (S)phenylethylcarbamate of amylose as a CSP and a mixture of hexane/EtOH/chloroform $60 / 20 / 20$ as the mobile phase.

\section{Supramolecular Structure of Polysaccharide Derivatives and Chiral Recognition Ability}

In the context of the project aiming to better understand the mechanism of interaction and chiral discrimination of small chiral molecules with polysaccharide derivatives, we conducted several investigations. The evidence of the importance of the supramolecular structure of microcrystalline cellulose triacetate in the chromatographic chiral discrimination process was already discussed in the first section of this review. It was also pointed out that this supramolecular structure is likely related to the molecular arrangement of the cellulose triacetate chains within the micro-crystals contained in the material. These domains offer a multitude of possible chiral cavities with varied spatial and electronic environments. ${ }^{[30]}$ Obviously, altering the crystal structure changes this environment and consecutively the chiral recognition properties of the material. It was also clear that a complete lack of crystallinity (amorphous material) is associated with a loss of chiral recognition ability. At this point, it must be emphasized that the importance of the supramolecular structure in the stereoselective interaction process implies that investigation of these interactions must occur in a state where this structure is not destroyed.

As discussed in a previous section, our observation that some molecules, in

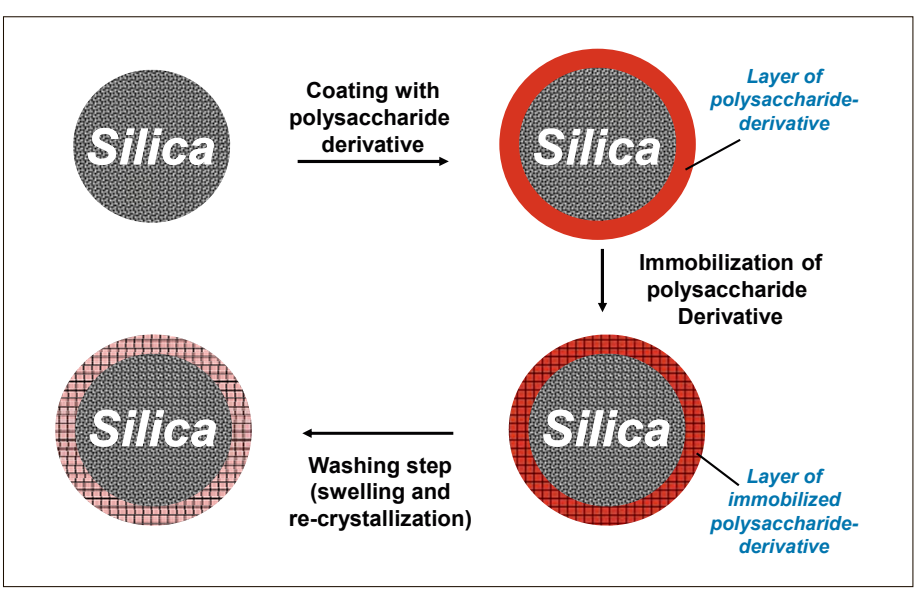

Fig. 21.

Immobilization concept for polysaccharide-based chira stationary phases.

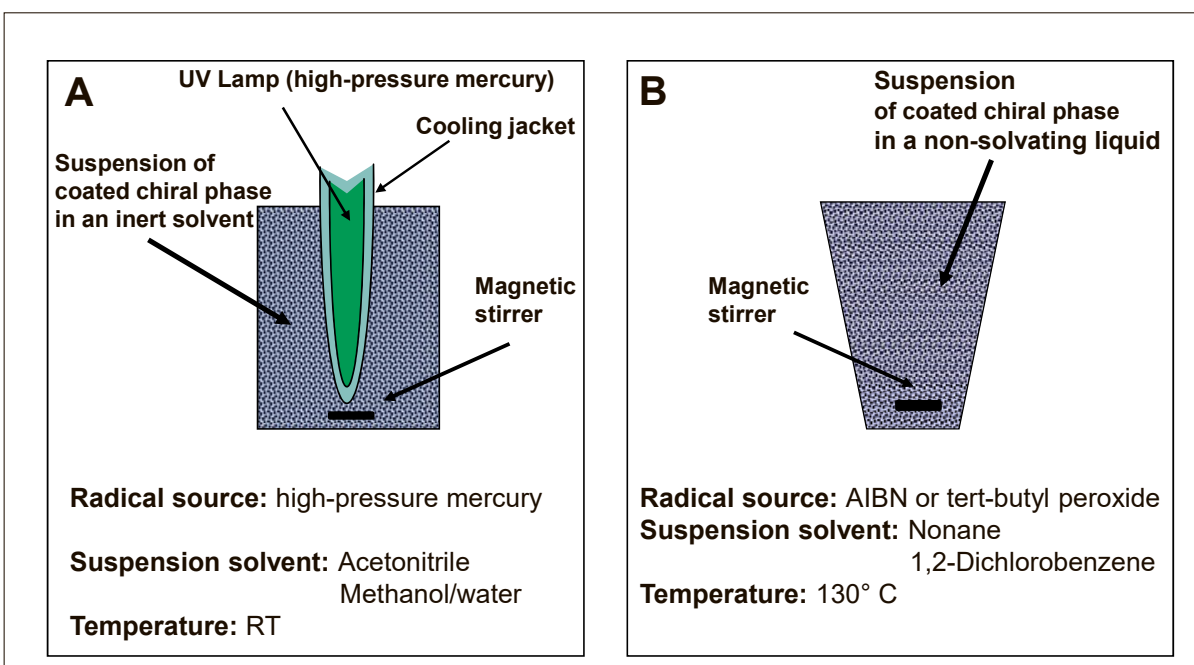

Fig. 22. Schematic sketch of the immobilization process for coated polysaccharide-based stationary phases.

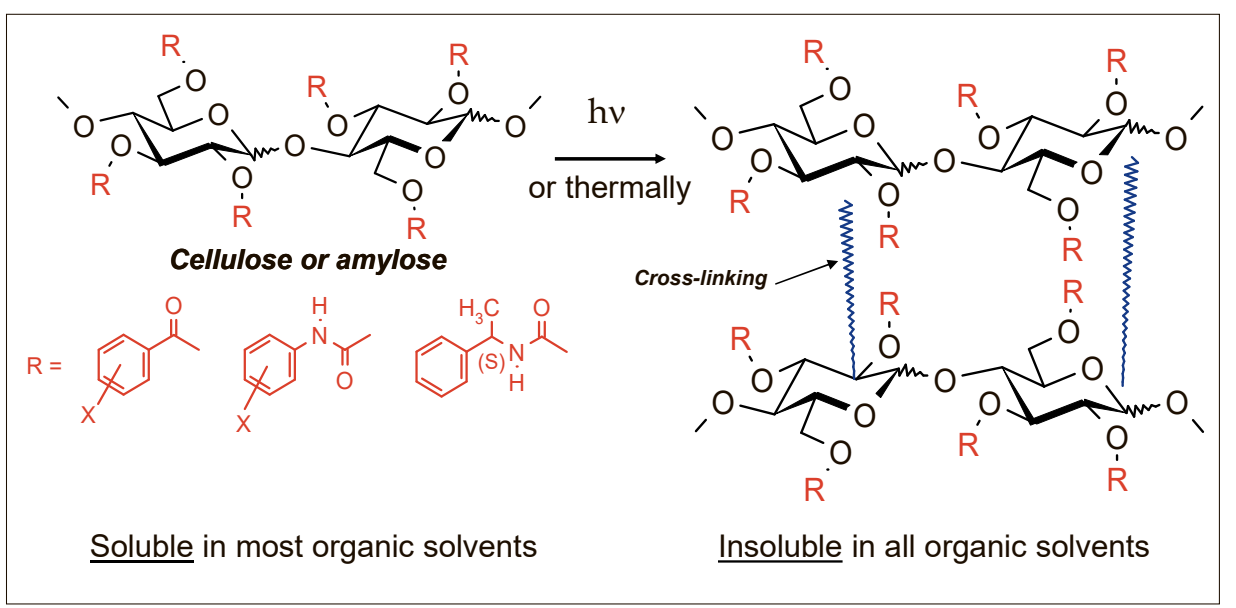

Fig. 23. Proposed mechanism for the immobilzation of coated polysaccharide-based stationary phases.

particular those containing a nitrophenyl group, are strongly retained on CTA $\mathrm{I}$, has led to the elaboration of a strategy of derivatization to enhance retention and improve selectivity for the separation of enantiomers. This strategy was very successful. Following on this observation we speculated that it might be possible to control the crystal packing of cellulose derivatives, by adding such strongly interacting substances during the coating process of polysaccharide derivatives on silica gel, the substances acting as a template mediating the pre-organization of an 
Table 3. Structures and immobilization process of the immobilized coated polysaccharides-based stationary phases

\section{Cellulose}

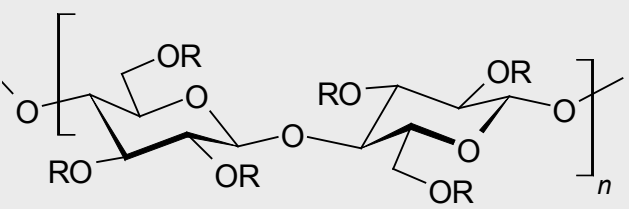

\section{Name}

Cellulose tribenzoate

Cellulose tris (4-methylbenzoate)

Cellulose tris (3-methylbenzoate)<smiles>CC(=O)c1cccc(C)c1</smiles>

Cellulose tris (phenylcarbamate)

Cellulose tris (4-methylphenylcarbamate)

Cellulose tris (3,5-dimethylphenyl carbamate)<smiles>CC(=O)Nc1ccccc1</smiles><smiles>CC(=O)Nc1ccc(C)cc1</smiles>

Cellulose tris (4-chlorophenylcarbamate)

Cellulose tris (3,4-dichlorophenylcarbamate)<smiles>CC(=O)Nc1cc(C)cc(C)c1</smiles><smiles>CC(=O)Nc1ccc(Cl)cc1</smiles><smiles>CC(=O)Nc1ccc(Cl)c(Cl)c1</smiles>

Cellulose tris (3,5-dichlorophenylcarbamate)<smiles>CC(=O)Nc1cc(Cl)cc(Cl)c1</smiles>

Cellulose tris (3-chloro-4methylphenylcarbamate)

Cellulose tris (3-trifluoromethyl-4chlorophenylcarbamate)

$$
\mathbf{R}=
$$<smiles>CC(=O)c1ccccc1</smiles><smiles>CC(=O)c1ccc(C)cc1</smiles><smiles>CC(=O)Nc1ccc(C)c(Cl)c1</smiles>

Thermal, Peroxide in nonane $130{ }^{\circ} \mathrm{C}$

$\mathrm{UV}$ in $\mathrm{MeOH} /$ water

Photochemical, UV (+ thioxanthone) in $\mathrm{MeOH} /$ water

Photochemical, Peroxide in nonane $130{ }^{\circ} \mathrm{C}$

Thermal, Peroxide in 1,2 dichlorobenzene

Or photochemical, UV in $\mathrm{MeOH} /$ water

Photochemical, UV in $\mathrm{MeOH} /$ water

Photochemical, UV in $\mathrm{MeOH} /$ water

Photochemical, UV in acetonitrile

Photochemical, UV in $\mathrm{MeOH} /$ water

Photochemical, UV in $\mathrm{MeOH} /$ water

\section{Thermal, Peroxide in nonane $130^{\circ} \mathrm{C}$}

C

Amylose

\section{Name}

Amylose tris [(s)-t-methylbenzylcarbamate]

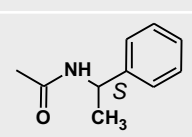

Amylose tris (3,5-dimethylphenylcarbamate) 
ordered structure of the polymer before it solidifies. We expected to exploit this feature to improve the chiral recognition ability of polysaccharide-based phases. This was investigated using meta-methylbenzoyl cellulose which was dissolved in different solvents in the presence or absence of additives before coating on silica gel. ${ }^{58-60] ~ M e t a-m e t h y l b e n z o y l ~ c e l l u l o s e ~}$ was adsorbed on silica gel using pure dichloromethane or mixture with tetra- hydrofurane or phenol. The same operation was performed with pure nitrobenzene and with a mixture of nitrobenzene with phenol. When a series of racemic compounds were chromatographed under the same conditions (mobile phase, flow rate, temperature) on columns packed with the obtained coated materials, it was observed i) that the enantioselectivity of the materials significantly varied, and ii) that for two compounds, an inversion of elution

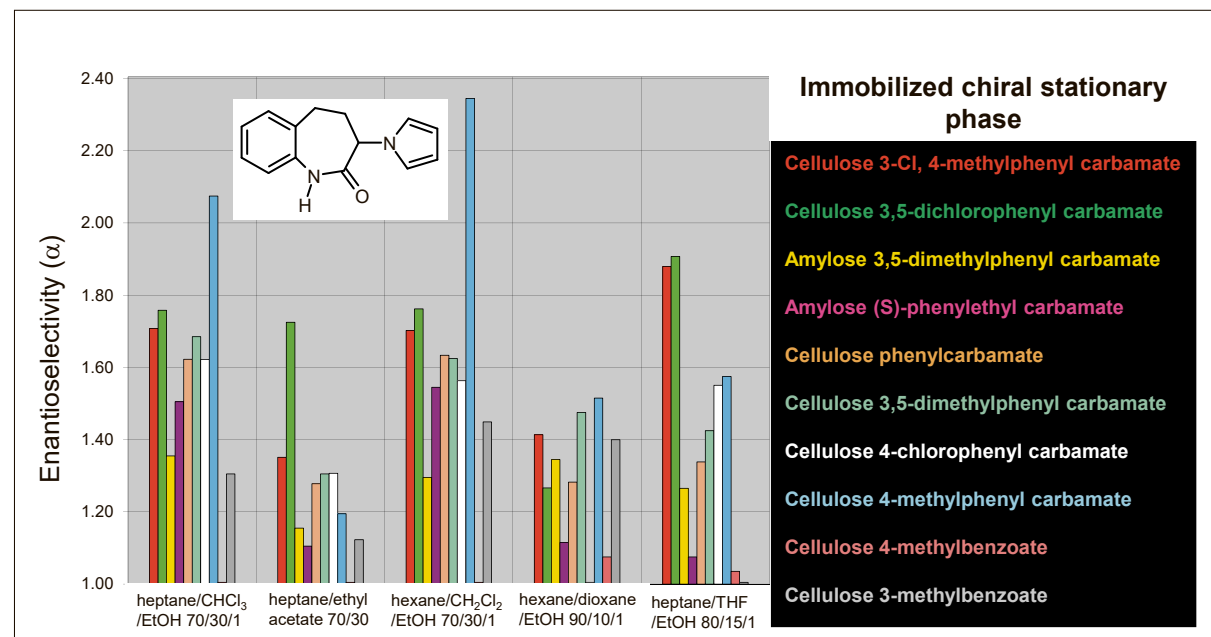

Fig. 24. Chiral recognition ability of immobilized polysaccharide-based CSPs. Influence of CSP type and mobile phase composition on enantioselectivity $(\alpha)$ for the separation of the enantiomers of a chiral tetrahydro-benzazepinone derivative. Colors of the bars in the diagram correspond the colors of the stationary phases.

order occurred if pure dichloromethane is used during the coating procedure (Table 4 and Fig. 28). This result demonstrated again that two chiral stationary phases made from the same chiral polysaccharide-based material, thus having the same chiral information at the molecular level can afford opposite chiral recognition ability for a particular chiral compound. This soundly suggests that two different supramolecular structures were produced, depending on the experimental conditions and that the supramolecular structure governs the chiral recognition process.

The way that the cellulose derivative is adsorbed on silica gel also greatly affects the chiral recognition ability of the chromatographic material. Table 5 shows the effect of 'precipitating' the polysaccharide derivative by evaporation of the solvent or by precipitation by adding a precipitant to such a poor solvent for the polysaccharide derivative. The 'precipitation' process clearly generally appears more effective, but there were some exceptions.

All results presented in this section, as well as those previously discussed for CTA, emphasize the difficulty to predict the effect of any 'operation' when preparing polysaccharide-based phases. Similar observations were made by Shibata et al. on CSPs made from cellulose triacetate and benzoate but no inversion of elution was reported.[61]

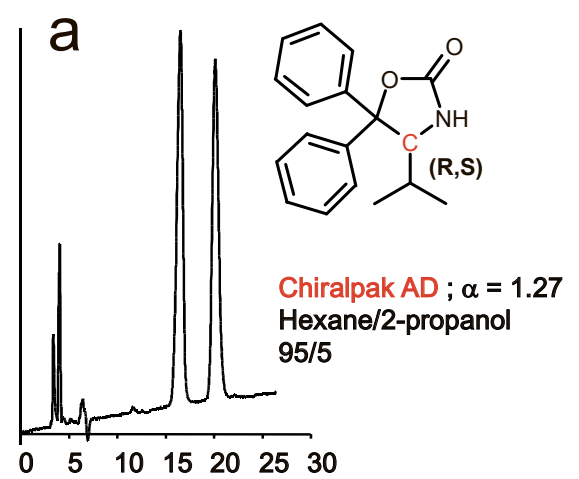

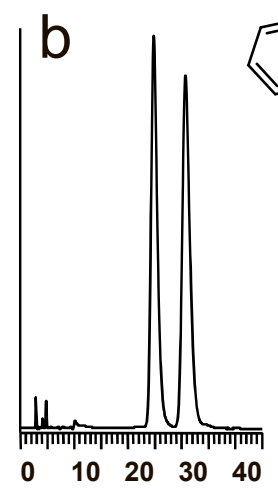

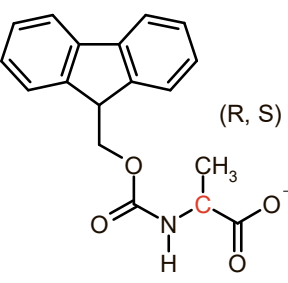

Chiralcel OD ; $\alpha=1.27$ Hexane/ethanol $90 / 10$

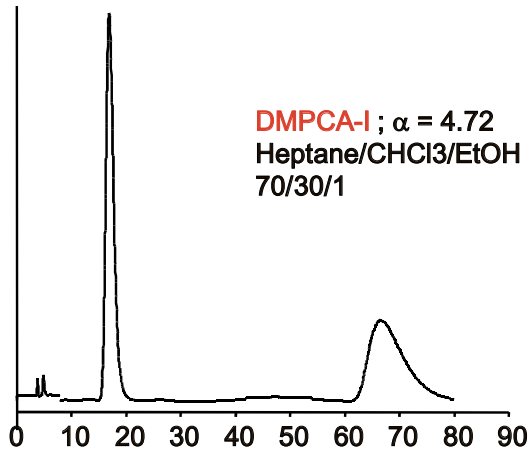

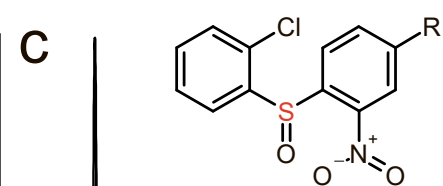

Chiralcel OD ; $\alpha=2.56$ Hexane/ethanol $30 / 70$
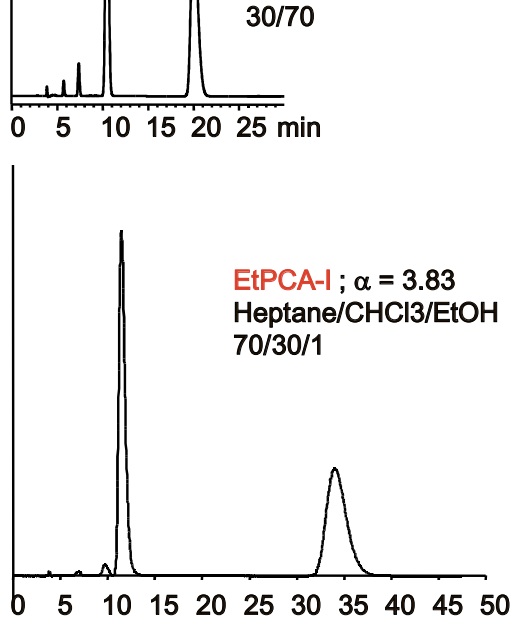

Fig. 25. Separations of enantiomers using immobilized polysaccharide-based phases and unusual mobile phases (bottom) versus non-immobilized phases (top): a) top on Chiralpak AD and bottom on immobilized 3,5-dimethyphenyl carbamate of cellulose (DMPCC-I); b) top on Chiralcel OD and bottom on immobilized 3,5-dimethyphenyl carbamate of amylose (DMPCA-I); c) top on Chiralcel OD and bottom on immobilized (S)ethylphenylcarbamate of amylose (EtPCA-I). Applied mobile phases and enantioselectivity are indicated on the respective chromatograms. 
Another approach to get more insight into the mechanism of interaction of cellulose-based CSPs with chiral molecules was followed by synthesizing and evaluating the chiral recognition power of a series of cellulose derivatives which were regioselectively derivatized on the 2,3 , and 6 positions of the glucose moiety. ${ }^{[59,60,62]}$ The objective was to determine whether some positions of the sugar moiety are 'more' or 'less' relevant for the chiral recognition process. For this purpose, 16 possible combinations with the hydroxyl groups in the 2,3 , and 6 positions being esterified with benzoyl, 2-methylbenzoyl, 3-methylbenzoyl, or 4-methylbenzoyl chloride were synthesized. In all combinations, positions 2 and 3 always carry identical groups, due the applied synthetic procedure (Fig. 29). The chromatographic material was prepared using the standard process by coating silica gel with the respective mixed cellulose esters.

Selected racemic compounds were injected on columns packed with the coated mixed esters. Table 6 shows the enantioselectivity obtained for four different racemic compounds, using hexane-ethanol $90 / 10$ as the mobile phase. Interestingly, for three compounds the best selectivity was obtained on one of the homogeneously substituted cellulose derivative, while one compound is better resolved on a mixed ester phase. From these results, it is difficult to draw conclusions about the role of the esters on the different positions of the glucose moiety, considering that no consistent relationship could be found between the substitution pattern of the mixed phases and their chiral recognition ability for all compounds. For example, glutethimide is well resolved on the phases carrying an ortho-methylbenzoate group on position 6, and does not 'feel' the effect of the groups in position 2 and 3 of the glucose unit. By contrast, 1-phenylethanol is well resolved on the homogeneously derivatized tribenzoylcellulose and 'feels' very much any change in positions 2 and 3 of the glucose unit. These unpredictable results again suggest the importance of the conformation of the polysaccharide chain and the resulting supramolecular arrangement of these chains, which are both very likely affected by the type of substituent on the glucose moiety. Although the purpose of this study was rather mechanistic, it was interesting to notice that mixed phases might exhibit a higher selectivity for some compounds. However, in practice it does not make much sense to develop so many different phases.

In conclusion, the determining influence of the supramolecular structure of polysaccharide derivatives on their chiral recognition ability is obviously a fascinating topic of discussion and it may explain the high versatility of this type of chiral

stationary phases. However, it emphasizes also the importance of carefully controlling all parameters related to the preparation of the stationary phases. It stresses also the difficulty of modeling and predicting chi-

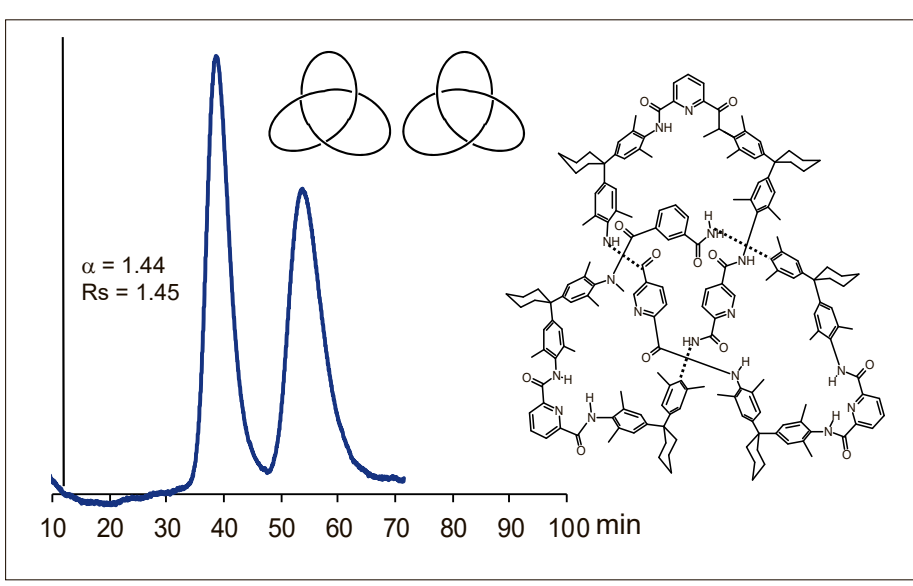

Fig. 26. Separation of the enantiomers of a chiral knot on immobilized 3,5-dimethylphenylcarbamate of cellulose. Column, $0.46 \mathrm{~cm} \times 25 \mathrm{~cm}$, mobile phase, heptane/ chloroform/ethanol $65 / 30 / 5$

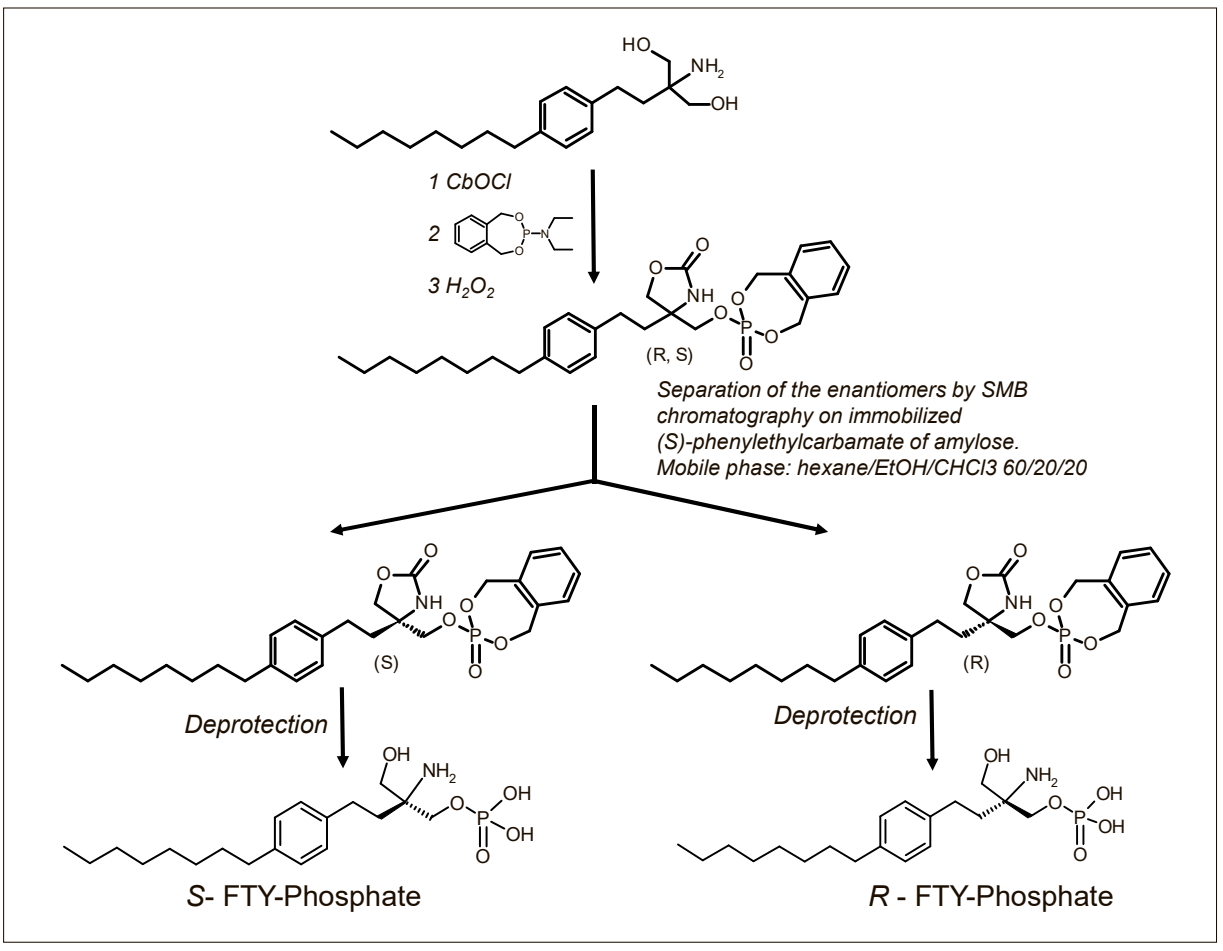

Fig. 27. Process of preparation of the enantiomers of the phosphate derivatives of the drug Fingolimod.

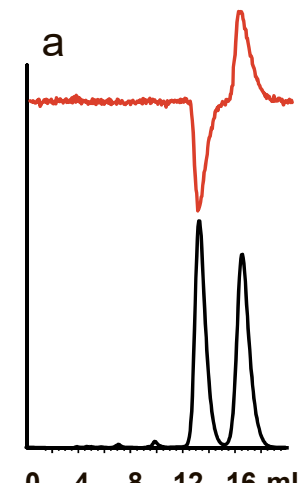

$\begin{array}{lllll}0 & 4 & 8 & 12 & 16 \mathrm{ml}\end{array}$
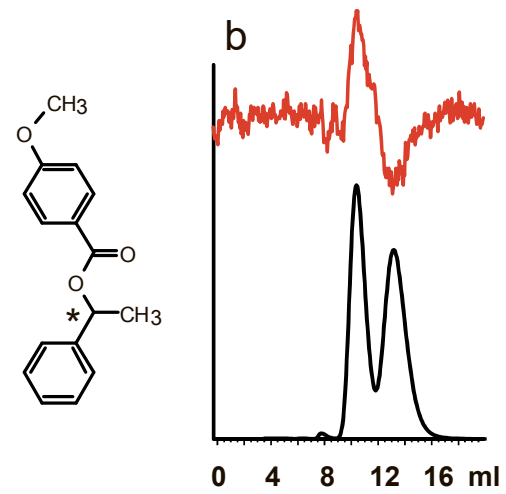

Fig. 28. Influence of coating conditions on chiral recognition ability of silica-coated 3-methylbenzoyl cellulose: a) Coating by precipitation from dichloromethane; b) Coating by precipitation from nitrobenzene 
Table 4. Influence of coating solvent on chiral recognition ability of silica-coated meta-methylbenzoyl cellulose. Enantioselectivity ( $\alpha$ ) and elution order (sign) of first eluted enantiomer are given in the Table. Column, $0.4 \mathrm{~cm} \times 25 \mathrm{~cm}$; mobile phase, hexane/2-propanol 9/1; flow rate, 0.7 ml/min.

\begin{tabular}{|c|c|c|c|c|c|}
\hline \multirow[t]{2}{*}{ Coating solution } & $\begin{array}{l}\text { Methylene } \\
\text { chloride }\end{array}$ & $\begin{array}{c}\text { Methylene } \\
\text { chloride-THF } \\
2.54 / 1\end{array}$ & $\begin{array}{l}\text { Methylene } \\
\text { Chloride/ } \\
\text { phenol 8/1 }\end{array}$ & $\begin{array}{l}\text { Nitrobenzene } \\
\text { /phenol } \\
8 / 1\end{array}$ & Nitrobenzene \\
\hline & \multicolumn{5}{|c|}{ Enantioselectivity $(\alpha)$} \\
\hline & $1.59(-)$ & $1.52(-)$ & $1.65(-)$ & $1.65(-)$ & $1.43(-)$ \\
\hline & $2.97(+)$ & $1.95(+)$ & $1.57(+)$ & $1.60(+)$ & $1.63(+)$ \\
\hline & $1.71(-)$ & $1.86(-)$ & $1.92(-)$ & $1.84(-)$ & $1.74(-)$ \\
\hline & $1.22(-)$ & $1.13(+)$ & $1.70(+)$ & $1.60(+)$ & $1.32(+)$ \\
\hline & $1.36(-)$ & $1.00(-)$ & $1.62(+)$ & $1.52(+)$ & $1.40(+)$ \\
\hline
\end{tabular}

cases of inversion of elution order when changing the mobile phase are common. Although this phenomenon has not yet been elucidated, we have speculated that the mobile phase might alter the supramolecular structure of the polymeric material due to a swelling effect which modifies the size of the interaction cavity between the polysaccharide chains. This swelling treatment is even necessary to 'activate' cellulose triacetate (CTA I) to obtain good selectivity and resolution performances. [13]

All these features of polysaccharidebased phases make the prediction of chiral separations very questionable and this remains, still today, a major challenge in the field, although this kind of phase is the most popular one and completely dominates the market for enantioselective separations.

\section{Special Applications}

The great success of polysaccharidebased materials as chiral stationary phases is attested by the numerous applications which are published every year in the field, although only a very limited number of works appear in the public domain. Based on the figures regarding the utilization of

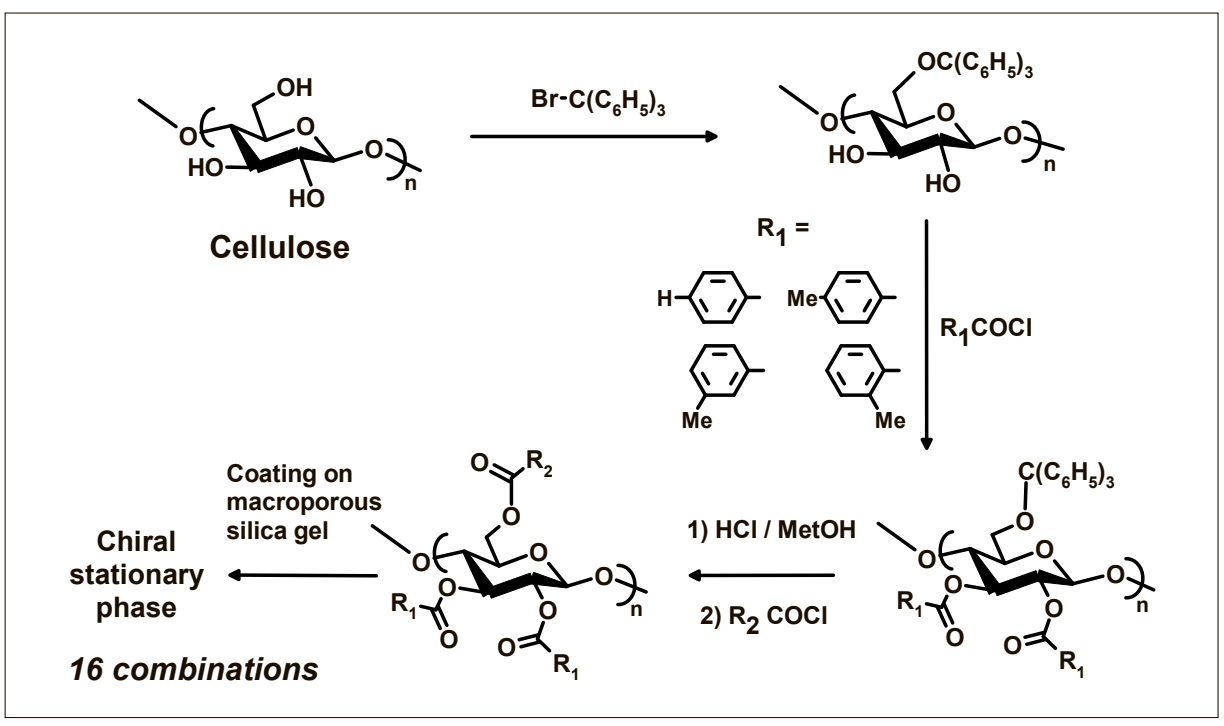

Fig. 29. Synthetic scheme for the preparation of regioselectively derivatized benzoylcellulose derivatives.

the technique in a large pharmaceutical company like Novartis, it can be estimated more than hundred thousand of new chiral molecules are analytically or preparatively resolved every year by enantioselective chromatography. With the currently available tools, it is possible to resolve almost all chiral molecules with any type of chirality. It would be unreasonable to list the applications which were performed in our laboratories over the last 30 years, but a few examples illustrating the power of enantioselective chromatography to investigate chiral molecules which would be 
difficult to examine by other methods are presented below.

Atrope isomers are a class of chiral molecules which is usually particularly challenging to resolve into the corresponding enantiomers. Enantioselective chromatography provides a very mild and broadly applicable tool for this type of chirality. It was particularly helpful to separate and investigate the biological activity of the single stereoisomers of a CCR5 antagonist for transplantation. ${ }^{[63]}$ As this molecule contains two axes of chirality, the separation of all four stereoisomers was a real challenge. The separation could be successfully achieved on the amylose-based phase Chiralpak AD. Additionally, the barrier to rotation around the chirality axis was relatively low, causing a rapid interconversion of the isomers at room temperature (Fig. 30), making their isolation for biological testing also difficult. By performing a micro-prep separation at lower temperature, sufficient amounts of the pure stereoisomers could be isolated and tested.

A second example of a chiral molecule with hindered rotation around the biphenyl bond is shown in Fig. 31. In this case, the molecule contains also a center of chirality, causing the presence of four stereosiomers. Enantioselective chromatography again was a powerful tool to discriminate the four stereoisomers. Moreover, it permitted to recognize the lability of the atropo isomerism, distinguishable from the presence of a plateau between the two enantiomers (for each pair), indicating the dynamic character of the isomerization. By heating up to $70{ }^{\circ} \mathrm{C}$, coalescence of the peaks reveals the rapid interconversion of the atrope isomers. This phenomenon can be used to experimentally calculate the barrier to rotation of atrope isomers. ${ }^{[64]}$ This example shows also the crucial influence of the mobile phase on chiral recognition. Changing the mobile phase composition from heptane/ ethanol 60/40 to heptane/ethanol/methanol $60 / 20 / 20$ causes an inversion of one pair of stereoisomers (Fig. 31).

In the context of the growing number of chiral molecules exhibiting atrope isomerism in pharmaceutical research and development, the usefulness of enantioselective chromatography for separating this kind of stereoisomers, which are otherwise often difficult to separate, has been particularly valued in the pharmaceutical field.

\section{Supercritical Fluid Chromatography and Continuous Chromatography for Enantioselective Separations}

The successful application of enantioselective chromatography was not solely due to the development of powerful and versatile chiral stationary phases but also to the concomitant development of technologies (Fig. 32).

Table 5. Influence of coating procedure on retention (capacity factor k1) and chiral recognition ability (enantioselectivity $\alpha$ ) of silica-coated meta-methylbenzoyl cellulose. Column $0.4 \mathrm{~cm} \mathrm{x}$ $25 \mathrm{~cm}$; mobile phase hexane/2-propanol 9/1; flow rate $0.7 \mathrm{ml} / \mathrm{min}$.

Coating procedure<smiles>CCC(Oc1ccc(Cl)cc1Cl)C(=O)c1cccnc1</smiles><smiles>CC1COc2ccccc2N1C</smiles><smiles>CC(=O)c1cc2c(cc1O)C(C(C)=O)CC2</smiles><smiles>COc1ccc(C(=O)OC(C)c2ccccc2)cc1</smiles><smiles>CC(C)(C(O)c1c2ccccc2cc2ccccc12)C(F)(F)F</smiles>

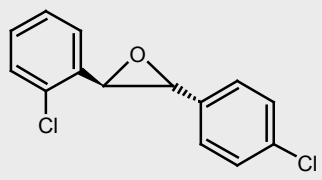

0.68

Deposition by precipitation

$\mathbf{k}_{1}$

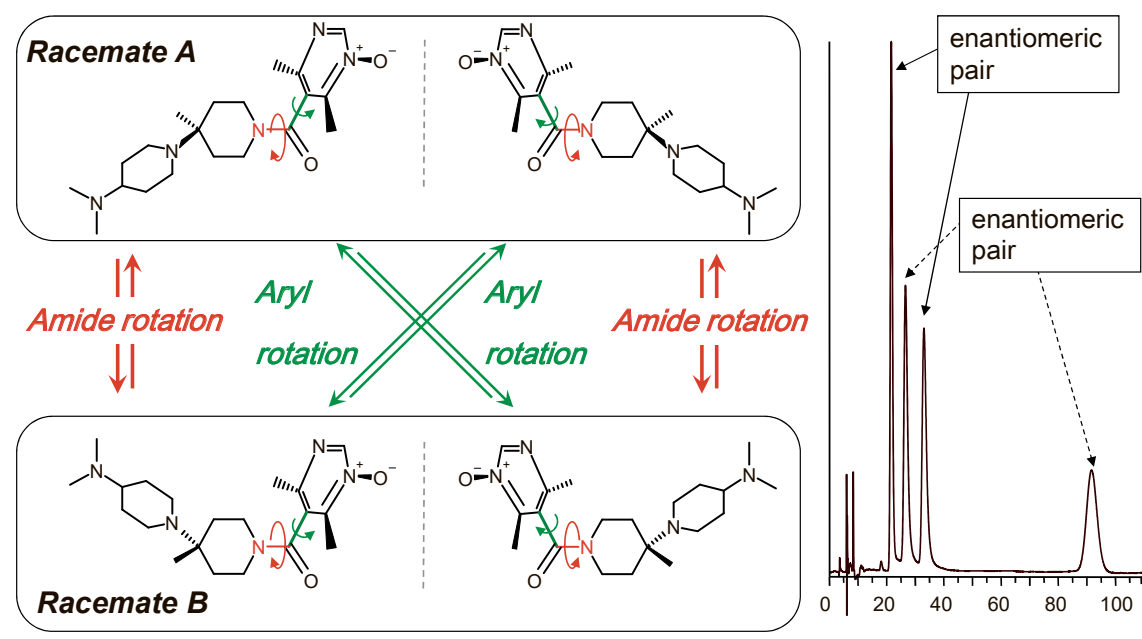

Fig. 30. Structures and separation of the atropisomers of a CCR5 antagonist drug for transplantation on Chiralpak AD. Column, $4.6 \times 25 \mathrm{~cm}$; mobile phase hexane/ethanol 15/85; flow rate, $1 \mathrm{ml} / \mathrm{min}$.

In drug discovery, rapid transfer from the analytical data to the preparative application was a major requirement, while in development or production, a continuous process was highly preferred. Working at the interface between discovery and development, we became very early interested in evaluating two technologies for the purpose of large-scale enantioselective separations. One technology was super- 
Table 6. Enantioselective separations on regioselectively substituted methylbenzoyl cellulose derivatives. Enantioselectivity obtained on the homogeneously substituted cellulose derivatives are highlighted in yellow.

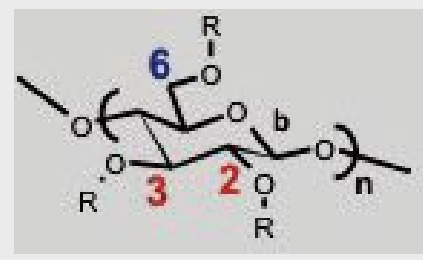

$\mathrm{R}$ in glucose position $6^{1)}$

$\mathrm{R}$ in glucose position $2,3^{1)}$
Benzoyl

\begin{tabular}{c|c}
$\begin{array}{c}\text { meta- } \\
\text { methyl- }\end{array}$ & para- \\
methyl- \\
benzoyl & benzoyl
\end{tabular}

ortho-

methyl-

benzoyl

Enantioselectivity $(\alpha)$

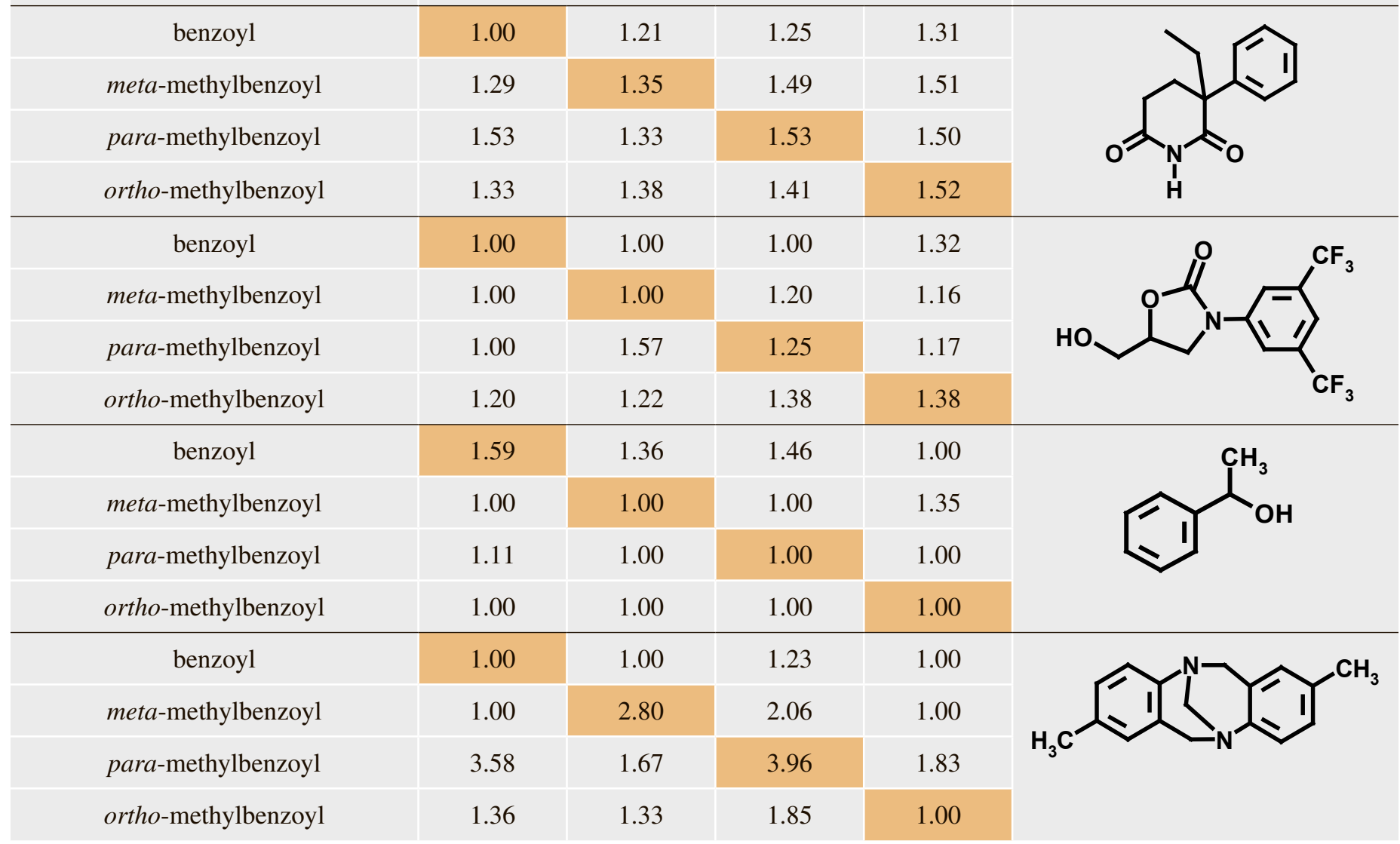

critical fluid chromatography (SFC) and the second one is called simulated moving bed chromatography (SMBC). These two technologies were known for a long time but were not applied in the pharmaceutical industry in the early nineties.

In 1992, we simultaneously started an internal project with chemical development at former Ciba-Geigy[65] and a collaboration with Prochrom in France to evaluate the potential of SFC for preparative separation of enantiomers. Independently of this SFC project, we acquired in 1994 a prototype of simulated moving bed equipment from Universal Oil Products (UOP). Both technologies were new for the pharmaceutical industry, and interestingly both were boosted by the very specific application of enantioselective chromatography. SFC which was a topic of interest since 1980 but declining in the early nineties has been 'revived' by the enantioselective chromatography application and SMBC has been introduced in the life science industry thanks to the enantioselective chromatography application.

SFC is basically no different from normal phase chromatography, which is the classical mode of chromatography for enantioselective separations. The main difference resides in the fact that the organic mobile phase is replaced to a large extent by carbon dioxide in its supercritical state, which is characterized by a very low viscosity. Supercritical carbon dioxide offers numerous advantages over classical normal phase HPLC. These advantages include high diffusivity due to the low viscosity, permitting low pressure drops, rapid chromatography, and short equilibration times. Besides these technical advantages, the reduced solvent consumption and costs, fewer safety concerns with respect to flammability and toxicity, reduced impact on the environment, and in preparative applications fast solvent removal make SFC a very attractive separation technique. Although SFC had many technical hurdles in the early stage of the development of the technique, it is now the preferred mode of chromatography in most research environments where chiral analysis and fast preparative resolution of racemic compounds are required. ${ }^{[66]}$ Fig. 33 shows a typical preparative chromatographic resolution of a chiral drug intermediate using the standard stacked injection procedure with very short cycle times. Since many years, the technique is applied daily at Novartis using almost exclusively polysaccharide-based material as chiral stationary phases.

In 1994, a 'new' technique called simulated moving bed chromatography 
(SMBC) has been made available for continuous purifications. Actually, the technique was not new and had been developed in the early sixties for very large scale purification in the petrochemical industry, but for more than 30 years nobody had thought that it could be beneficial in the pharmaceutical field for high added value substances. This has been made finally feasible by down-sizing the existing concept and the commercialization of equipment, which was developed more or less simultaneously by UOP (US) and by Novasep (France). During the same period of time, a few groups had built up their own homemade systems. ${ }^{[67-71]}$ Simulated moving bed chromatography is a continuous purification process which is particularly designed for purifying binary mixtures. Details about the principle of SMBC are available from many publications and reviews. ${ }^{[72]}$ Due to the nature of racemic substances (mixture of two enantiomers), it was inherent that the technology perfectly fit the requirements of large-scale separation of enantiomers. This stimulated us to invest in the technology and we acquired the instrument from UOP (first in Europe) which consisted of 16 columns $(6 \mathrm{~cm}$ by $2.1 \mathrm{~cm})$ arranged in series and contained in total about $200 \mathrm{~g}$ of chiral stationary phase (Fig. 34). The system was run in a closed setup, automatically and $24 \mathrm{~h}$ a day, delivering the separated enantiomers in high optical purity. ${ }^{[73]}$

In an early investigation productivity comparisons were performed with classical batch chromatography for two chiral drugs, the antiasthmatic substance Formoterol and the antitussive agent Guaifenesine. ${ }^{[73]}$ The study confirmed the much higher performance for SMB. Additionally, the solvent consumption was reduced by up to $90 \%$ using the SMB process. In a more detailed investigation in collaboration with Morbidelli and Mazzotti from the ETH Zurich, a more rationalized approach to optimize the operational SMB conditions was developed. ${ }^{[74]}$ The high performance of the technology and the possibility to process larger amounts of chiral compounds within a reasonable time frame rapidly led to an increasing demand and number of applications of SMB at Novartis research. Later, we acquired a larger SMB system from Bayer Technology Services (BTS). This system, which contained in total about $1 \mathrm{~kg}$ of stationary phase $(6 \mathrm{col}-$ umns 15 by $5 \mathrm{~cm}$ ), was capable to resolve up to several kilograms of enantiometric mixtures per day. Hundreds of chiral drugs or drug intermediates were separated on a large scale in support of medicinal chemistry research and of chemical development, but most applications were not published for confidential reasons. In all instances, polysaccharide-based phases were used as chiral stationary phases.

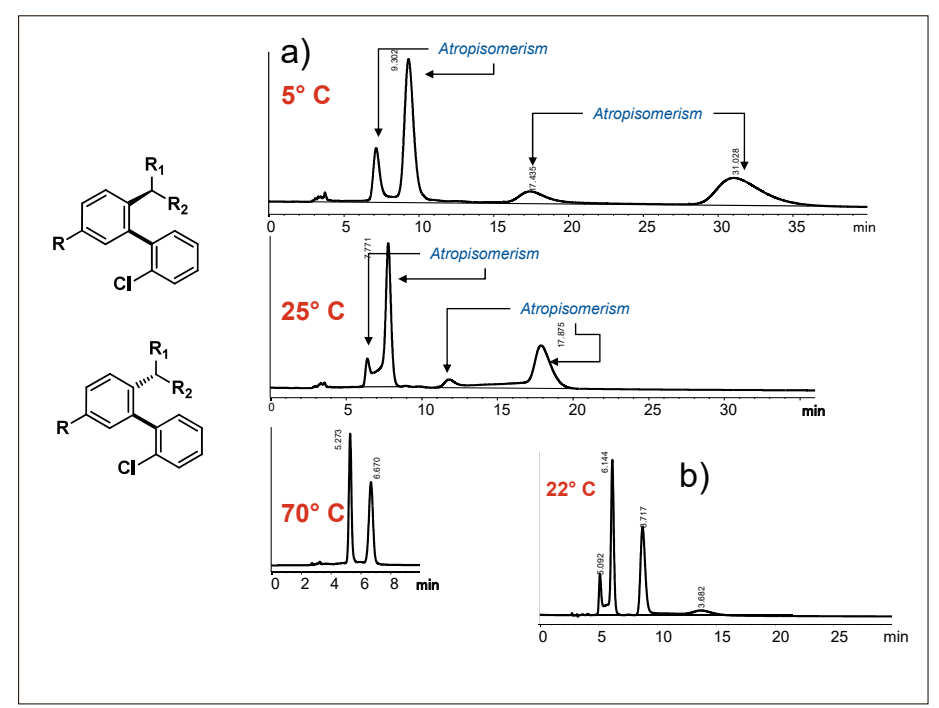

Fig. 31. Influence of temperature on the separation of the four stereoisomers of a compound containing a chirality axis and a chiral carbon atom. Column: Chiralcel OD $(4.6 \times 25 \mathrm{~cm})$; flow rate: $1 \mathrm{ml} / \mathrm{min}$; a) mobile phase: heptane/EtOH 60/40; b) heptane/EtOH/ $\mathrm{MeOH} 60 / 20 / 20$.

CSPs (Chemistry)

- Chirally modifidied carriers

- Chiral polymers (natural, synthetic)

- Imprinted materials

\section{Separation technologies}

- Modern HPLC

- SFC

- SMB, SSR, Varicol

- $C P C$

- CE, MEKC

- Parallel screening HPLC/SFC

Fig. 32. Chiral stationary phases and chromatographic techniques.

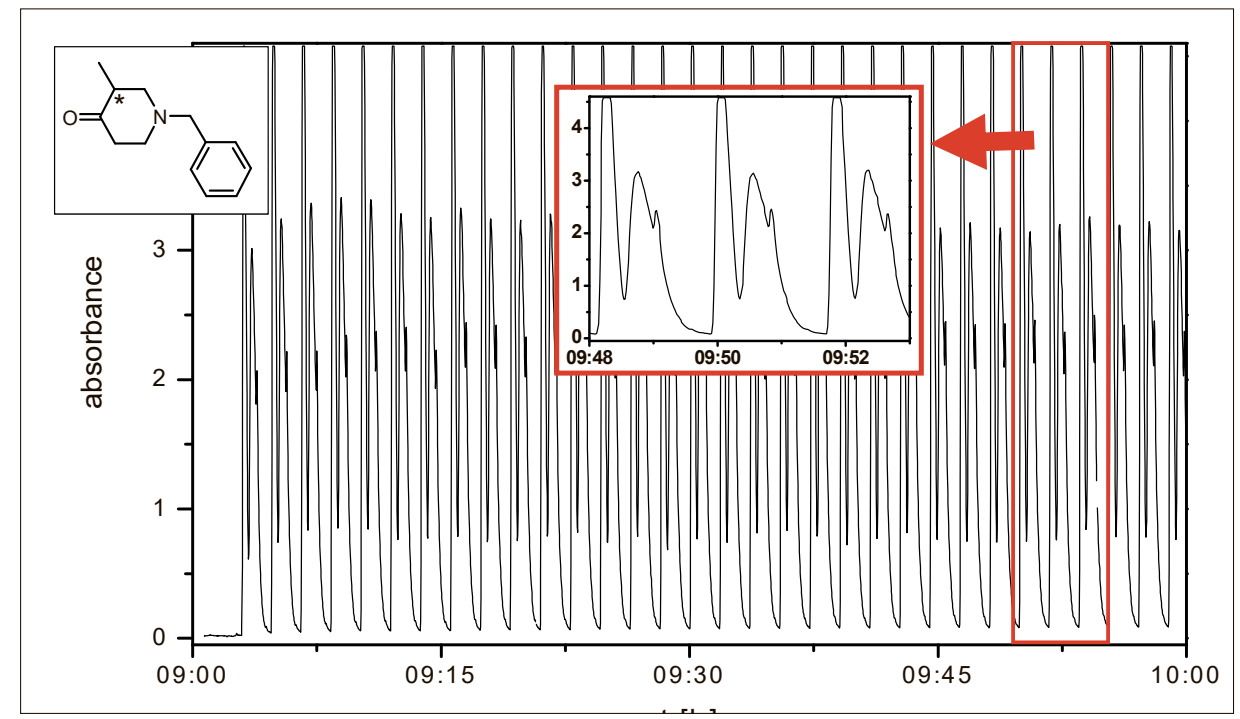

Fig. 33. Preparative separation of the enantiomers of $50 \mathrm{~g}$ of the building block 1-N-benzyl-3methyl-4-piperidinone by SFC. Column: Chiralpak AD-H, $5 \mu \mathrm{m}, 30 \mathrm{~mm} \times 250 \mathrm{~mm}$; mobile phase: CO2/EtOH 95:5; $120 \mathrm{~g} / \mathrm{min} ; 240$ stacked injection (à $208 \mathrm{mg}$ ) in $7 \mathrm{~h} 22 \mathrm{~min}$; cycle time: $1.8 \mathrm{~min}$

Among the published applications, it is worthwhile to mention the application to tert-leucine as a chiral building block $^{[75]}$ and some contributions to the development of several new chiral drugs at Novartis, such as the antiasthmatic agent Formoterol,[73] a new antimalarial agent, ${ }^{[76]}$ Fingolimod for the treatment of multiple sclerosis, ${ }^{[56,57]}$ 3,5-disubstituted piperidine building blocks for medicinal chemistry, ${ }^{[77]}$ a hypolipidemic agent, ${ }^{[78]}$ the anti-cancer agent aminoglutethimide, ${ }^{[73]}$ and many other building blocks (Fig. 35). The applications were in the range of a few hundreds of grams up to several kilograms. 
In several cases, we took advantage of the possibility to racemize the undesired enantiomer which could then be recycled, finally getting almost quantitatively one single enantiomer, starting from a racemic mixture. Racemization is usually achieved by basic or acidic treatment for molecules containing a chiral carbon atom but in the case of atropisomers, heating the molecules at higher temperature was sufficient. A typical practical example is shown in Fig. 36.

The concomitant advent of the SMB technology and the powerful polysaccharide-based chiral stationary phases has become a unique success story. It has permitted to considerably accelerate the development of new chiral drugs in the pharmaceutical industry and made possible the development of competitive processes to access these new drugs up to production scale. This success has also changed the global perception about chromatographic processes. It is now fully accepted in the industry and the utilization of SMBC has extended to further areas, in particular biological purification processes.

\section{Epilog}

When I started to work on the project of enantioselective chromatography 35 years ago in the central research laboratories of Ciba, there was no chromatographic column packed with chiral material available on the market. The usual method of determination of the optical purity of chiral substances was optical rotation, NMR with chiral solvating agents and HPLC of diastereomeric derivatives. Today, what was thought to be impossible only 30 years ago is considered routine. There are a broad variety of 'chiral' columns on the market and the earlier analytical methods for chiral compounds have been almost totally replaced by the chromatographic technique which is much more accurate and easy to operate. Nowadays, almost any type of chiral molecule can be directly resolved into the corresponding enantiomers without prior derivatization. It applies to all types of chirality (centro, axial, planar) and all types of chiral atoms (carbon, sulfur, phosphor, nitrogen, selenium, metal ...). Moreover, enantioselective chromatography has proved to be a powerful approach to resolve chiral substances on a preparative scale. In medicinal chemistry, it has advantageously replaced the often tedious synthetic approach, at least at the early discovery stage which required only small amounts of optically pure enantiomers. The chromatographic method is not only generally applicable, relatively simple and rapid, but it permits the two pure enantiomers to be obtained in one operation. This is obviously important at a stage where the biological activity of the individual enantiomers is not known. Even large scale separation of enantiomers by chromatography has become a standard approach for providing optically pure drugs or drug intermediates at a scale of a few hundreds of grams to kilograms. But what was totally unexpected is that the technique would be used at the production scale of several metric tons per year. There are now several chiral drugs on the market which have been 'produced' by enantioselective chromatography.

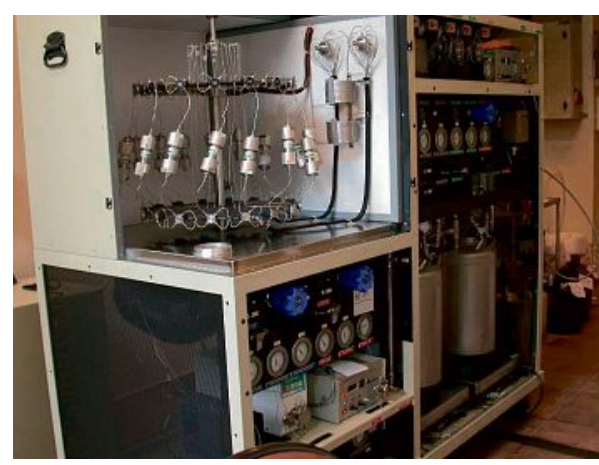

Fig. 34. Picture of the UOP simulated moving bed instrument (1994).

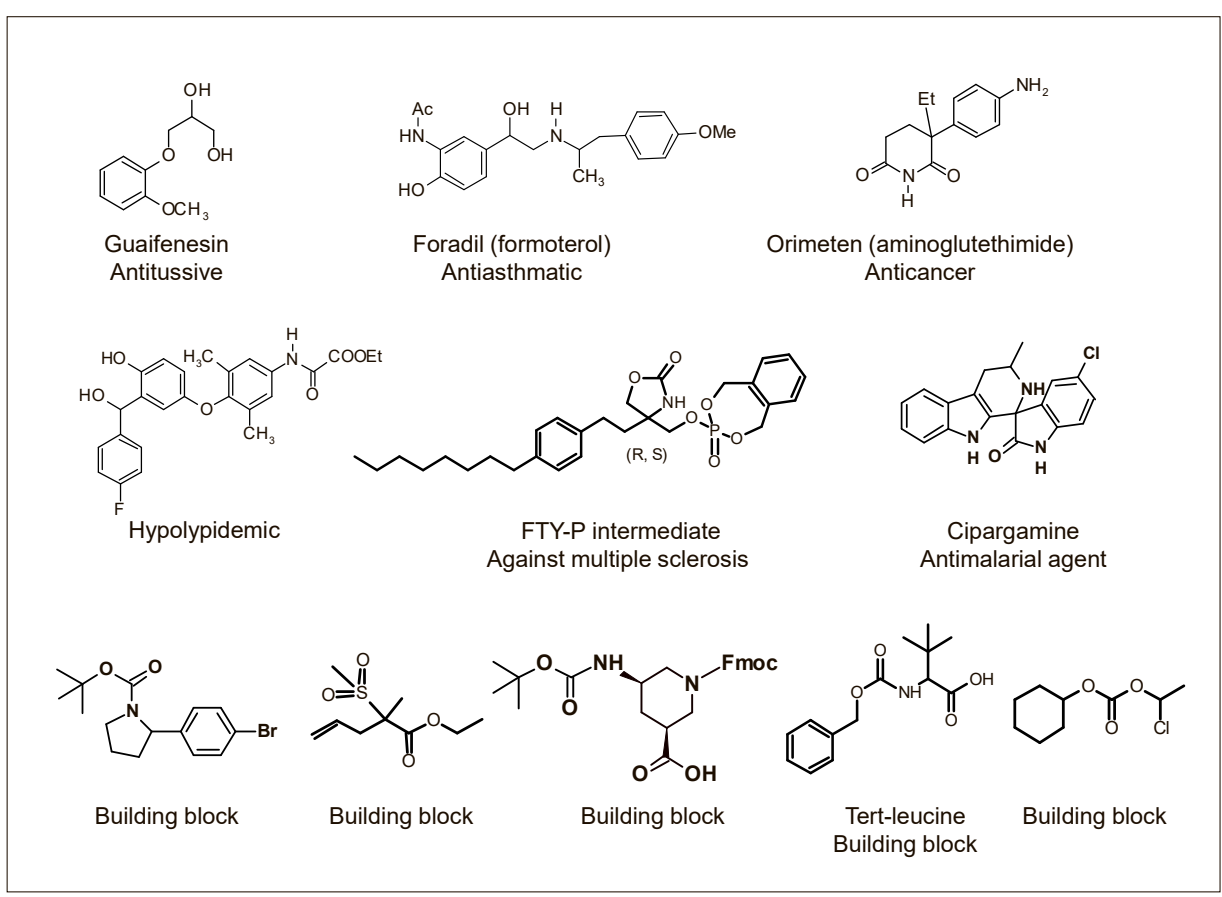

Fig. 35. Structures of selected racemic compounds resolved on a large scale by SMB chromatography.

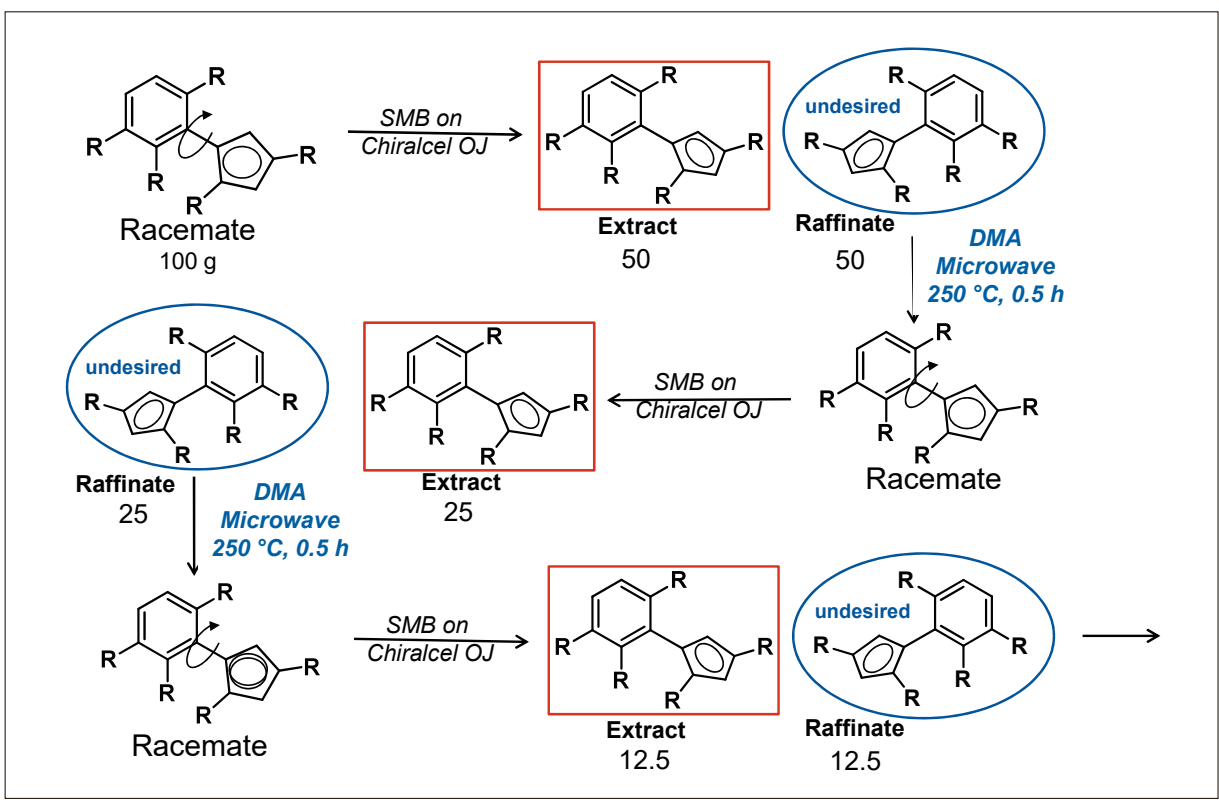

Fig. 36. Example of recycling of undesired enantiomer in a large scale resolution by SMB chromatography. 
With the project 'optically active polymers for enantioselective chromatography', Ciba-Geigy had very early recognized the importance of the relationship between chirality and biological activity, and thanks to the successful development of the technique, the company was for many years a step ahead of the competition. The development of immobilized polysaccharide-based phases was certainly a highlight of the story and it is rewarding to see that these phases are now the gold standard in the field. They are used by almost all laboratories dealing with chiral molecules in academia and industry across the world. In particular in the pharmaceutical industry, they greatly facilitate and make safer the development of new drugs.

This project, which was at the interface between chemistry, physics and engineering, was also a captivating adventure in the fascinating world of chirality.

\section{Acknowledgments}

This review describes the results of a long and intensive collaboration with many colleagues and friends over more than 35 years. The list is very long and I wish to thank all who contributed to the success of this project, most notably, Romain Wolf, Gabrielle Baisch, Marie-Luz Picollotto, Paul Richert, Dan Huynh, Gabrielle Lecis and Tong Zhang. I wish also to thank all the people who all along the years supported the project by trusting the team and providing the means to reach this great achievement, Roland Darms, Friedrich Lohse, Georges Haas, Dieter Lohmann, Rudolf Andreatta, Rene Amstutz, and Thomas Leutert. I would like to thank also Richard Lewis for providing modeled structures of polysaccharide derivatives.

Received: June 22, 2017

[1] G. M. Henderson, H. G. Rule, Nature 1938, 141, 917.

[2] V. Prelog, P. Wieland, Helv. Chim. Acta 1944, 27, 1127.

[3] S. V. Rogozhin, V. A. Davankov, J. Chem. Soc. $D$ 1971, 490.

[4] G. Blaschke, Angew. Chem., Int. Ed. Engl. 1971, $10,520$.

[5] E. Gil-Av, B. Feibush, R. Charles-Sigler, Tetrahedron Lett. 1966, 7, 1009.

[6] H. Yuki, Y. Okamoto, I. Okamoto, J. Am. Chem. Soc. 1980, 102, 6356.

[7] W. H. Pirkle, D. L. Sikkenga, J. Chromatogr. 1976, $123,400$.

[8] Amstrong. T. Yubing, Y. Zhou, D. Armstrong, $J$. Chromatogr. A 1994, 666, 147.

[9] V. Schurig, J. Chromatogr. A 1994, 666, 111.

[10] W. A. König, in 'The practice of Enantiomer Separation by Capillary Gas Chromatography', Hüthig, Heidelberg, 1992.

[11] W. Lindner, Chimia, 1981, 35, 204.

[12] K. Hess, N. Ljubitsch, Ber. dtsch. Chem. Ges. $A / B$ 1928, 61, 1460

[13] G. Hesse, R. Hagel, Liebigs Ann. Chem. 1976, 996.

[14] E. Francotte, R. M. Wolf, D. Lohmann, R. Müller, J. Chromatogr. 1985, 347, 25.
[15] E. Francotte, T. Zhang, J. Chromatogr. A, 1995, $718,257$.

[16] E. Francotte, R. M. Wolf, Chirality 1990, 2, 16.

[17] E. Francotte, H. Stierlin, J. W. Faigle, J. Chromatogr. 1985, 346, 321.

[18] E. Francotte, D. Lohmann, Helv. Chim. Acta 1987, 70, 1569

[19] H.-G. Capraro, E. Francotte, B. Kohler, G. Rihs, P. Schneider, R. Scartazzini, O. Zak, W. Tosch, J. Antibiot. 1988, 41, 759 .

[20] E. Francotte, R. W. Lang, T. Winkler, Chirality 1991, 3, 177.

[21] R. O. Duthaler, A. Hafner, M. Riediker, Pure Appl. Chem. 1990, 62, 631.

[22] E. Francotte, P. Ackermann, in 'Chirality and biological activity', Symposium book, Alan R. Liss, Inc., 1990, p. 63-70. [23] E Francotte, P. Ackermann, Eur. Pat. EP 145661 A2, Nov. 9, 1984.

[24] E. Francotte, A. Junker-Buchheit, $J$ Chromatogr. A 1992, 576, 1.

[25] E. Francotte, J. Chromatogr. A 1994, 666, 565.

[26] E. Francotte, in 'Chiral Separations: Applications and Technology', Ed. S. Ahuja, ACS Books, USA, 1996, Chap. 10, p. 271-308.

[27] E. Francotte, in 'The Impact of Stereochemistry on Drug Development and Use', Eds. H. Y. Aboul-Enein, I. W. Wainer, John Wiley \& Sons. Chemical Analysis Series, 1997, Vol. 142, Chap. 23, p. 633-683.

[28] I. Agranat, H. Caner, J. Caldwell, Nature Rev. Drug Dis. 2002, 1, 753.

[29] W. Eckhardt, E. Francotte, J. Herzog, P. Margot, G. Rihs, W. Kunz, Pestic. Sci. 1992, 36, 223.

[30] R. M. Wolf, E. Francotte, D. Lohmann, J. Chem Soc. Perkin Trans. II 1988, 893.

[31] R. M. Wolf , E. Francotte, J. Hainmüller, Chirality 1993, 5, 538.

[32] E. Francotte, G. Baisch: European Patent, EP 0316270 A2, 1989 (Case, 6-16740; Priority, October 26, 1987).

[33] E. Francotte, R. M. Wolf, Chirality 1991, 3, 43 .

[34] E. Francotte, Patent WO 9627639 A1 (Case 4-20361/P1; 7.3.1995).

[35] Y. Okamoto, M. Kawashima, K.Yamamoto, K. Hatada, Chem. Lett. 1984, 13, 739

[36] Y. Okamoto, M. Kawashima, K. Hatada, J. Am. Chem. Soc. 1984, 106, 5357.

[37] E. Yashima, C. Yamamoto, Y. Okamoto, Synlett, 1998, 344.

[38] E. Francotte and R. M. Wolf, J. Chromatogr. 1992, 595, 63.

[39] E. Francotte, Proceedings of the ChiralEurope ' 96 Symposium, 1996, 89.

[40] J. Drabek, E. Francotte, D. Lohmann, F. Bourgeois, Ger. Pat. DE 4000335 A1, Jan. 11, 1989.

[41] P. Furet, C. Batzl, A. Bhatnagar, E. Francotte, G. Rihs, M. Lang, J. Med. Chem. 1993, 36, 1393.

[42] I. Ernest, J. Kalvoda, C. Sigel, G. Rihs, H. Fritz, M. J. Blommers, F. Raschdorf, E. Francotte, M. Mutter, Helv. Chim. Acta 1993, 76, 1539.

[43] S. Drenkard, J. Ferris, A. Eschenmoser, Helv. Chim. Acta, 1990, 73, 1373.

[44] A. Huxley-Tencer, E. Francotte, M. BladochaMoreau, Pestic. Sci. 1992, 34, 65.

[45] E. Francotte, Chirality, 1998, 10, 492.

[46] E. Francotte, T. Zhang, International Patent number WO 1997/04011(06.22.1997); European Patent EP 0840752B1 (priority 21.07.1995).

[47] E. Francotte, International Patent number WO 1997/049733(31.12.1997); European Patent EP 0907663B1 (priority 27.06.1996).

[48] E. R. Francotte, Chimia 1997, 51, 717.

[49] E. Francotte, J. Chromatogr. A 2001, 906, 379.

[50] E. Francotte, D. Huynh, J. Pharm. Biomed. Anal. 2002, 27, 421.

[51] H. Wetli, E. Francotte, J. Sep. Sci. 2007, 30, 1255.

[52] E. Francotte, D. Huynh, T. Zhang, Molecules 2016, 21, 1740.
[53] J. Shen, T. Ikai, Y. Okamoto, J. Chromatogr. A 2014, 1363, 51

[54] C. Amoss, G. Cox, P. Franco, T. Zhang, Chiral Technologies, Application Note, http://chiraltech.com/technical-library/\#applicationnotes

[55] Chiral knot: F. Vogtle, A. Hunten, E. Vogel, S. Buschbeck, O. Safarowsky, J. Recker, A.-H. Parham, M. Knott, W. Müller, U. Müller, Y. Okamoto, T. Kubota, W. Lindner, E. Francotte, S. Grimme, Angew. Chem., Int. Ed. 2001, 40, 2468.

[56] A. Rainer, E. Francotte, F. Zecri, M. Zollinger, Patent WO 2005021503 (Case 33329 P1), 2005.

[57] R. Albert, K. Hinterding, V. Brinkmann, D Guerini, C. Mueller-Hartwieg, H. Knecht, C. Simeon, M. Streiff, T. Wagner, K. Welzenbach, F. Zecri, M. Zollinger, N. Cooke, E. Francotte, $J$. Med. Chem. 2005, 48, 5373.

[58] E. Francotte, T. Zhang, J. Chromatogr. A 1995, $718,257$.

[59] E. R. Francotte, T. Zhang, Analusis 1995, 23, M13.

[60] E. R. Francotte, T. Zhang, GIT Fachz. Lab. 1996, 40, 15 .

[61] T. Shibata, T. Sei, H. Nishimura, Chromatographia 1987, 24, 552.

[62] Lecture at the 19th International Symposium on Liquid Chromatography, HPLC' 95, Innsbruck (Austria), May 28- June 2, 1995.

[63] G. Thoma, F. Nuninger, M. Schaefer, H.G. Akyel, R. Albert, C. Beerli, C. Bruns, E. Francotte, M. Luyten, D. MacKenzie, L. Oberer, M.B. Streiff, T. Wagner, H. Walter, G. Weckbecker, H.-G. Zerwes, J. Med. Chem. 2004, 47, 1939.

[64] O. Trapp, V. Schurig, Computers and Chemistry 2001, 25, 187.

[65] K. Anton. K. Anton, J. Eppinger, E. Francotte, Poster presentation at the 'Supercritical Chemistry Symposium', Nottingham (UK), 1993.

[66] 'Supercritical Fluid Chromatography', Ed. C. Poole, Handbooks in Separation Science series, Elsevier, Amsterdam, ISBN: 978-0-12-809207$1,2017$.

[67] R.-M. Nicoud, LC-GC Intl. 1992, 5, 43.

[68] E. Küsters, F. D. Antia, Chromatographia 1995, 40, 387.

[69] E. Cavoy, M.-F. Deltent, S. Lehoucq, D. Miggiano, J. Chromatogr. 1997, 769, 49.

[70] M. Negawa, F. Shoji, J. Chromatogr. 1992, 590, 113.

[71] C.B. Ching, B.G. Lim, E.J.D. Lee, S.C. Ng, J. Chromatogr. 1993, 215, 113

[72] M. Juza, M. Mazotti, M. Morbidelli, Trends Biotech. 2000, 18, 108.

[73] E. Francotte, P. Richert, J Chromatogr A 1997, $769,101$.

[74] E. Francotte, P. Richert, M. Mazotti, M. Morbidelli, J. Chromatogr. A 1997, 796, 101.

[75] E. Francotte, T. Leutert, L. La Vecchia, F. Ossola, P. Richert, A. Schmidt, Chirality 2002, 14,313 .

[76] B. K. S. Yeung, B. Zou, M. Rottmann, S. B. Lakshminarayana, S. H. Ang, S. Yong Leong, J. Tan, J. Wong, S. Keller-Maerki, C. Fischli, A. Goh, E. Schmitt, P. Krastel, E. Francotte, K. Kuhen, D. Plouffe, K. Henson, T. Wagner, E. A. Winzeler, F. Petersen, R. Brun, V. Dartois, T. T. Diagana, T. H. Keller. J. Med. Chem. 2010, 53, 5155.

[77] J. C. D. Hartwieg, J. W. Priess, H. Schütz, D Rufle, C. Valente, C. Bury, L. La Vecchia, E. Francotte, Org. Process Res. Dev. 2014, 18,1120 .

[78] T. K. Majumdar, L. L. Martin, D. Melamed, F.L.S. Tse, J. Pharm. Biomed. Anal. 2000, 23, 745. 